\title{
Direct organocatalytic Wittig/Hetero-Diels-Alder reactions in one-pot: synthesis of highly-substituted tetrahydropyranones
}

\author{
Dhevalapally B. Ramachary,* Rumpa Mondal, and Sangeeta Jain \\ Catalysis Laboratory, School of Chemistry, University of Hyderabad, \\ Central University (PO), Hyderabad-500 046, India \\ E-mails: ramsc@uohyd.ernet.in, ramchary.db@gmail.com
}

\section{Dedicated to Prof. Dr. J. S. Yadav in appreciation of his outstanding contributions to synthetic organic chemistry}

DOI: http://dx.doi.org/10.3998/ark.5550190.p009.252

\begin{abstract}
A practical and environmentally friendly organocatalytic one-pot strategy designed to furnish the hetero-Diels-Alder products was shown to be effective in the preparation of disubstituted tetrahydropyranones in a highly selective manner. (S)-1-(2-pyrrolidinylmethyl)pyrrolidine catalyzed an asymmetric assembly reaction involving a hetero-Diels-Alder reaction between alkylidene- and arylidene-acetones generated in situ from Wittig reactions with diethyl ketomalonate to furnish the substituted tetrahydropyranones in moderate to very good yields with moderate enantioselectivity.
\end{abstract}

Keywords: Amino acids; diethyl ketomalonate; hetero-Diels-Alder reactions; multicomponent reactions; organocatalysis

\section{Introduction}

Cycloaddition reactions between carbonyl compounds as the heterodienophiles and homodienes provide for the preparation of numerous six-membered oxygen-containing heterocycles that are frequently encountered structural motifs in biologically active natural products. $^{1-3}$ The extraordinary range of applications of enantio-pure hetero-Diels-Alder (HDA) adducts has stimulated the search for efficient chiral catalysts for cycloadditions between dienes and carbonyl dienophiles. Many catalysts, including chiral aluminium, boron, titanium, chromium, europium, or ytterbium complexes, can accelerate the reaction of unactivated aldehydes with activated dienes to generate high yields with excellent stereochemical control. $^{4-7}$

Recently metal-free, small chiral organic molecules have emerged as an exciting class of biomimetic catalysts for the generation of enviromentally-friendly asymmetric reactions. ${ }^{8-10}$ Rawal et al. recently demonstrated that small organic molecules, such as TADDOL 
(tetraaryl-1,3-dioxolane-4,5-dimethanol), could catalyze the enantioselective HDA reaction of activated dienes with aldehydes through hydrogen bonding. ${ }^{11-13}$ Also, Jørgensen and Juhl developed the enantioselective inverse-electron-demand HDA reaction with an enamine generated in situ from an aldehyde and chiral amine with enones under organocatalysis. ${ }^{14-18}$ As part of our program to engineer novel asymmetric assembly reactions that proceed in environmentally-sound conditions under amine-catalysis, ${ }^{8,19-27}$ we sought to extend the use of chiral organo-amines as catalysts for asymmetric HDA reactions.

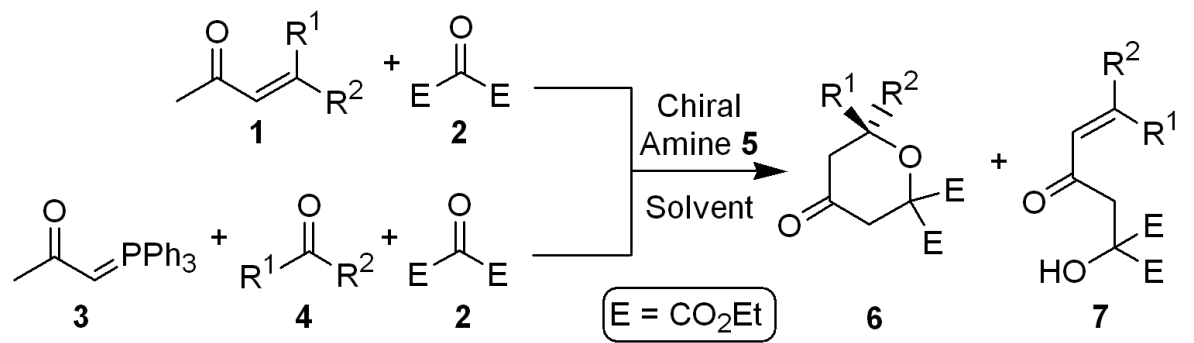

1a: $\mathrm{R}^{1}=\mathrm{H}, \mathrm{R}^{2}=\mathrm{C}_{6} \mathrm{H}_{5} ; 1 \mathrm{~b}: \mathrm{R}^{1}=\mathrm{H}, \mathrm{R}^{2}=4-\mathrm{OHC}_{6} \mathrm{H}_{4} ; 1$ c: $\mathrm{R}^{1}=\mathrm{H}, \mathrm{R}^{2}=$ 2,6- $\mathrm{Cl}_{2} \mathrm{C}_{6} \mathrm{H}_{3} ;$ 1d: $\mathrm{R}^{1}=\mathrm{H}, \mathrm{R}^{2}=$ trans- $\mathrm{CH}=\mathrm{CHPh} ; \mathbf{1 e}: \mathrm{R}^{1}=\mathrm{H}, \mathrm{R}^{2}=$ trans- $\mathrm{CH}=\mathrm{CHCH}_{3} ;$ 1f: $\mathrm{R}^{1}=\mathrm{H}, \mathrm{R}^{2}=\mathrm{CH}_{3} ; \mathbf{1 g}: \mathrm{R}^{1}=\mathrm{H}, \mathrm{R}^{2}=\mathrm{C}_{3} \mathrm{H}_{7} ; \mathbf{1 h}: \mathrm{R}^{1}=\mathrm{H}, \mathrm{R}^{2}=$ $\mathrm{C}_{4} \mathrm{H}_{9} ; 1 \mathrm{i}: \mathrm{R}^{1}=\mathrm{H}, \mathrm{R}^{2}=\mathrm{C}_{5} \mathrm{H}_{11} ; \mathbf{1 j}: \mathrm{R}^{1}=\mathrm{R}^{2}=\mathrm{CH}_{3} ; \mathbf{1 k}: \mathrm{R}^{1}=\mathrm{H}, \mathrm{R}^{2}=4-\mathrm{MeOC}_{6} \mathrm{H}_{4}$; 1I: $R^{1}=H, R^{2}=4-\mathrm{FC}_{6} \mathrm{H}_{4} ; 1 \mathrm{~m}: \mathrm{R}^{1}=\mathrm{H}, \mathrm{R}^{2}=4-\mathrm{ClC}_{6} \mathrm{H}_{4} ; 1 \mathrm{n}: \mathrm{R}^{1}=\mathrm{H}, \mathrm{R}^{2}=$ 4- $\mathrm{BrC}_{6} \mathrm{H}_{4} ; 10: \mathrm{R}^{1}=\mathrm{H}, \mathrm{R}^{2}=4-\mathrm{CNC}_{6} \mathrm{H}_{4} ; 1 \mathrm{p}: \mathrm{R}^{1}=\mathrm{H}, \mathrm{R}^{2}=4-\mathrm{NO}_{2} \mathrm{C}_{6} \mathrm{H}_{4}$

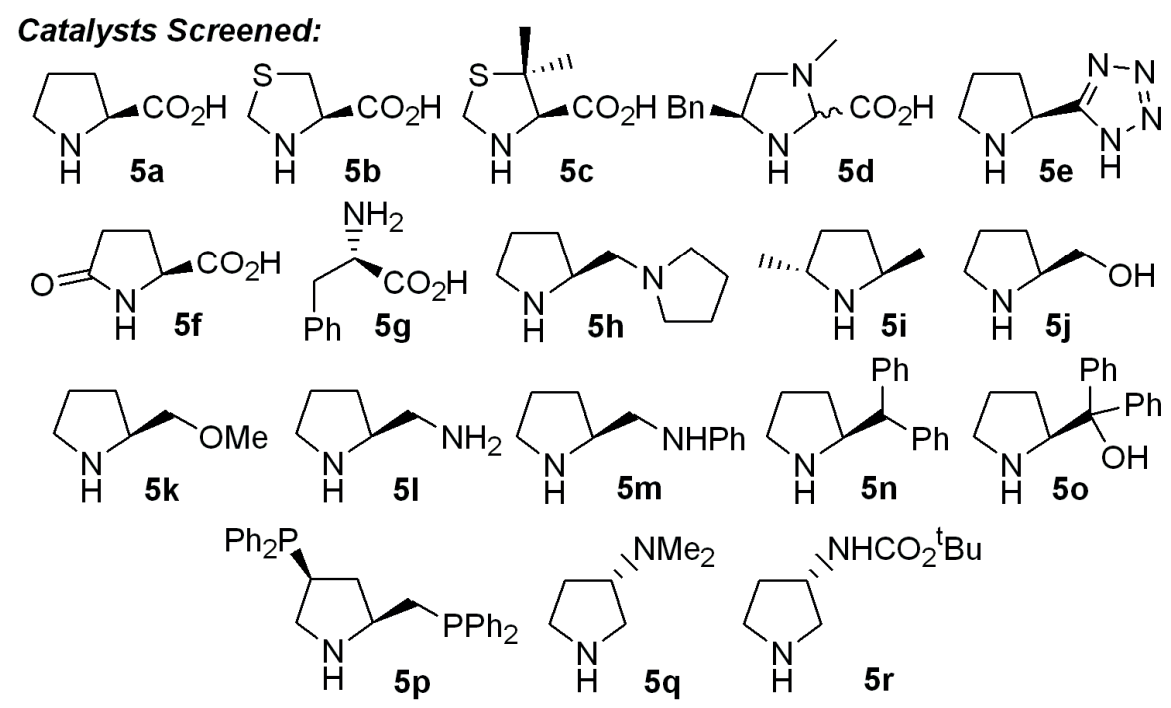

Scheme 1. Direct organocatalytic asymmetric Wittig/hetero-Diels-Alder reactions in one pot.

In contrast to cycloadditions involving aldehydes as the heterodienophiles, the DielsAlder reactions of ketones are still a challenge to chemists. Ketones are less reactive than aldehydes due to both steric and electronic constraints. The challenge herein is to develop a metal-free, small organic molecule-catalyzed, enantioselective HDA reaction of ketones with in situ generated conjugated dienes as this reaction will have wide utility in organic chemistry. In this article, we demonstrate the two- and three-component HDA reaction of in situ generated Barbas dienamines (2-amino-1,3-butadienes) 10 from enones 1 (generated from Wittig reaction of aldehydes 4 with 1-(triphenylphosphanylidene)propan-2-one $\mathbf{3}$ ) and 
chiral organo-amines $\mathbf{5}$ with activated ketone $\mathbf{2}$ as heterodienophile to furnish chiral disubstituted tetrahydropyranones $\mathbf{6}$ in good yields with moderate enantioselectivity as shown in Scheme 1. These HDA products 6 have direct application in the total synthesis of antiosteoporotic and antibiotic natural products. ${ }^{28-30}$

\section{Results and Discussion}

The reaction of benzylideneacetone 1a and diethyl ketomalonate $\mathbf{2}$ with a catalytic amount of (S)-1-(2-pyrrolidinylmethyl)pyrrolidine $\mathbf{5 h}$ in $\mathrm{MeOH}$ at ambient temperature for $17 \mathrm{~h}$ furnished the HDA product $\mathbf{6 a}$ and the aldol product 7a at a 7:1 ratio respectively, in 99\% yield but with only $3 \%$ enantiomeric excess (ee) of $\mathbf{6 a}$ (Table 1 , entry 1$)$.

Table 1. Effect of solvent on direct organocatalytic HDA reactions of $\mathbf{1 a}$ and $\mathbf{2}^{a}$

\begin{tabular}{ccccc} 
& & & \\
\hline & & & \\
\hline
\end{tabular}

${ }^{a}$ Reactions were carried out in solvent $(1 \mathrm{~mL})$ with $0.5 \mathrm{mmol}$ of 2 and $1.0 \mathrm{mmol}$ of $1 \mathrm{a}$ in the presence of $20 \mathrm{~mol} \%$ of catalyst. ${ }^{b}$ Yield refers to the column-purified product. ${ }^{c}$ Ratio determined by ${ }^{1} \mathrm{H}$ NMR analysis. ${ }^{d}$ Ee determined by CSP-HPLC analysis. ${ }^{\mathrm{e}}$ Reaction time was $17 \mathrm{~h}$.

In the asymmetric HDA reaction of enone 1a and diethyl ketomalonate $\mathbf{2}$ catalyzed by diamine $\mathbf{5 h}$, we found that the solvent had a significant effect on reaction path, yields, and $e e$ 's of $6 \mathbf{a}$ and $7 \mathbf{a}$ (Tables 1 and 2). The HDA reaction catalyzed by diamine $\mathbf{5 h}$ produced products 6a and 7a with an excellent ratio of $6 \mathbf{a}$ to $7 \mathbf{a}$ and excellent yields, but poor ee's in protic solvents (Table 1, entry 1; Table 2, entries 2-6) and with poor chemoselectivity, good yields, and moderate ees in aprotic polar/nonpolar solvents (Table 1, entries 2-12). The same 
reaction in the ionic liquid, [bmim] $\mathrm{BF}_{4}$ provided $\mathbf{6 a}$ in $31 \%$ yield, albeit with low ee of $8 \%$ (Table 1, entry 4). More of the undesired aldol product 7a was formed in aprotic solvents compared to protic solvents as shown in Tables 1 and 2.

Table 2: Effect of protic solvents on the direct diamine $\mathbf{5 h}$ catalyzed HDA reactions of $\mathbf{1 a}$ and $\mathbf{2}^{a}$

\begin{tabular}{|c|c|c|c|c|c|}
\hline \multirow[b]{2}{*}{ Entry } & \multirow{2}{*}{ Solvent } & \multicolumn{2}{|c|}{$\begin{array}{c}\begin{array}{c}\text { Diamine } 5 \mathrm{~h} \\
(20 \mathrm{~mol} \%)\end{array} \\
\stackrel{\text { Solvent }(1 \mathrm{M})}{\mathrm{RT}, 8 \mathrm{~h}} \\
\mathrm{E=} \mathrm{CO}_{2} \mathrm{Et}\end{array}$} & $>\overbrace{6 a}^{P h}$ & \multirow[t]{2}{*}{$\mathrm{ee}(\%)^{d}$} \\
\hline & & Yield (\% & & Ratio $(6 \mathbf{a} / 7 \mathbf{a})^{c}$ & \\
\hline $1^{\mathrm{e}}$ & THF & 6 & & $1: 1.5$ & - \\
\hline 2 & $\mathrm{MeOH}$ & 82 & & $7.6: 1$ & 3 \\
\hline 3 & EtOH & 69 & & $9: 1$ & 6 \\
\hline 4 & $n$-PrOH & 63 & & $10.7: 1$ & 5 \\
\hline 5 & $i$-PrOH & 30 & & $9: 1$ & 12 \\
\hline 6 & $n$-ВuOH & 66 & & 12.8:1 & 2 \\
\hline 7 & $s e c-\mathrm{BuOH}$ & 5 & & 1:99 & - \\
\hline 8 & tert-BuOH & 5 & & 1:99 & - \\
\hline
\end{tabular}

${ }^{a}$ Reactions were carried out in solvent $(1 \mathrm{~mL})$ with $0.5 \mathrm{mmol}$ of 2 and $1.0 \mathrm{mmol}$ of $1 \mathrm{a}$ in the presence of $20 \mathrm{~mol} \%$ of catalyst. ${ }^{b}$ Yield refers to the column-purified product. $^{c}$ Ratio determined by ${ }^{1} \mathrm{H}$ NMR analysis. ${ }^{d}$ Ee determined by CSP-HPLC analysis. ${ }^{e}$ Reaction time was $17 \mathrm{~h}$.

Next we probed the structure and reactivity relationships among a family of 18 pyrrolidine-based catalysts by monitoring the reaction conversions and ee values of the HDA reaction of $\mathbf{1 a}$ and $\mathbf{2}$ in toluene (Table 3). The amino acid L-proline 5a catalyzed the HDA reaction to produce $\mathbf{6 a}$ and $7 \mathbf{a}$ in $45 \%$ conversion and $13 \%$ ee with $1: 1.4$ ratio of $\mathbf{6 a}$ to $7 \mathbf{a}$ after 5 days (Table 3, entry 1). L-Thiaproline $\mathbf{5 b}$ also catalyzed the HDA reaction to produce 6a and $7 \mathbf{a}$ in $20 \%$ conversion and $40 \%$ ee with $1: 2$ ratio of $6 \mathbf{a}$ to $7 \mathbf{a}$ after 5 days (Table 2, entry 2). Among the catalysts screened, $(S)$-2-diphenylmethylpyrrolidine $5 \mathbf{n}$ proved to be the most efficient catalyst with respect to ee, providing $6 \mathbf{a}$ in $49 \%$ ee but conversion $(30 \%)$ and ratio of $6 \mathbf{a}$ to $7 \mathbf{a}$ were poor (Table 3 , entry 14). Notable improvement in the enantioselectivity of the reaction was found in L-thiaproline- and $(S)$-2-diphenylmethyl-pyrrolidine-catalyzed HDA reactions.

Table 3. Effect of catalyst on the direct amine $\mathbf{5}$ catalyzed HDA reactions of $\mathbf{1 a}$ and $\mathbf{2}^{a}$

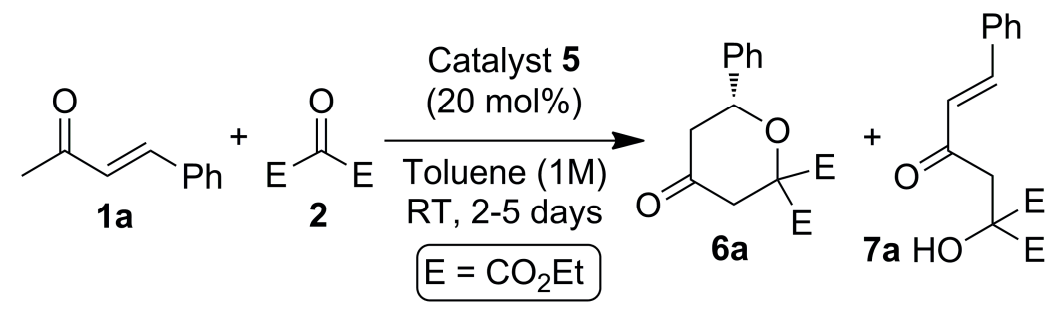


Table 3 (continued)

\begin{tabular}{ccccc}
\hline Entry & Catalyst & $\begin{array}{c}\text { Conversion } \\
(\%)^{b}\end{array}$ & ${\text { Ratio }(\mathbf{6 a} / \mathbf{7 a})^{c}}$ & ee $(\%)^{d}$ \\
\hline $1^{e}$ & $\mathbf{5 a}$ & 45 & $1: 1.4$ & 13 \\
2 & $\mathbf{5 b}$ & $\mathbf{2 0}$ & $\mathbf{1 : 2}$ & $\mathbf{4 0}$ \\
3 & $\mathbf{5 c}$ & $<10$ & - & - \\
4 & $\mathbf{5 d}$ & 15 & $1: 19$ & - \\
5 & $\mathbf{5 e}$ & 40 & $1: 1$ & 15 \\
6 & $\mathbf{5 f}$ & 60 & $1: 19$ & - \\
7 & $\mathbf{5 g}$ & 20 & $1: 19$ & - \\
$8^{f}$ & $\mathbf{5 h}$ & 65 & $5: 1$ & 5 \\
9 & $\mathbf{5 i}$ & 30 & $1: 9$ & - \\
10 & $\mathbf{5 j}$ & 60 & $2: 1$ & 8 \\
11 & $\mathbf{5 k}$ & 60 & $3: 1$ & 8 \\
12 & $\mathbf{5 1}$ & 75 & $5: 1$ & 15 \\
13 & $\mathbf{5 m}$ & $<10$ & - & - \\
$\mathbf{1 4}$ & $\mathbf{5 n}$ & $\mathbf{3 0}$ & $\mathbf{1}$ & $\mathbf{4 9}$ \\
15 & $\mathbf{5 0}$ & 10 & $1: 9$ & - \\
16 & $\mathbf{5 p}$ & $<10$ & - & - \\
17 & $\mathbf{5 q}$ & 30 & $1: 5$ & 7 \\
18 & $\mathbf{5 r}$ & 75 & $5: 1$ & 3 \\
\hline
\end{tabular}

${ }^{a}$ Reactions were carried out in toluene $(1 \mathrm{~mL})$ with $0.5 \mathrm{mmol}$ of 2 and $1.0 \mathrm{mmol}$ of $1 \mathrm{a}$ in the presence of $20 \mathrm{~mol} \%$ of catalyst 5 at RT for 2-5 days. ${ }^{b}$ Conversion based on the ratio of $\mathbf{1 a}$ to 6a and 7a, determined by ${ }^{1} \mathrm{H}$ NMR analysis. ${ }^{c}$ Ratio determined by ${ }^{1} \mathrm{H}$ NMR analysis. ${ }^{d} \mathrm{Ee}$ determined by CSP-HPLC analysis. ${ }^{e}$ Solvent was MeOH. ${ }^{f}$ Additive $\mathrm{CF}_{3} \mathrm{CO}_{2} \mathrm{H}(30 \mathrm{~mol} \%)$ was used.

The proposed mechanism for synthesis of chemoselective tetrahydropyranone $6 \mathbf{a}$ and aldol product 7a through the reaction of enone 1a and ketone $\mathbf{2}$ is illustrated in Scheme 2. Chiral (S)-1-(2-pyrrolidinylmethyl)pyrrolidine $\mathbf{5 h}$ or L-proline 5a presumably catalyze the in situ generation of 2-amino-1,3-butadiene $\mathbf{1 0}$ or 2-hydroxy-1,3-butadiene $\mathbf{8}$ from enone $\mathbf{1 a}$. Subsequent [4+2]-cycloaddition of $\mathbf{1 0}$ with ketone $\mathbf{2}$ furnishes the enamine 11, which immediately undergoes hydrolysis to furnish HDA product $\mathbf{6 a}$ or to aldol product $7 \mathbf{a}$ via retro-Michael reaction under protic solvent conditions. Alternatively, 2-hydroxy-1,3butadiene 8 reacts with ketone $\mathbf{2}$ to furnish aldol product 7a, which does not undergo intramolecular oxy-Michael addition to form $\mathbf{6 a}$ under these conditions. Interconversion of products $\mathbf{6 a}$ and 7a was confirmed by controlled experiments as shown in Scheme 3 (see Supporting Information). Formation of 2-hydroxy-1,3-butadiene 8, responsible for the generation of aldol byproduct 7a (see Scheme 3), is more favorable in aprotic nonpolar solvents under general acid/base-catalysis. 


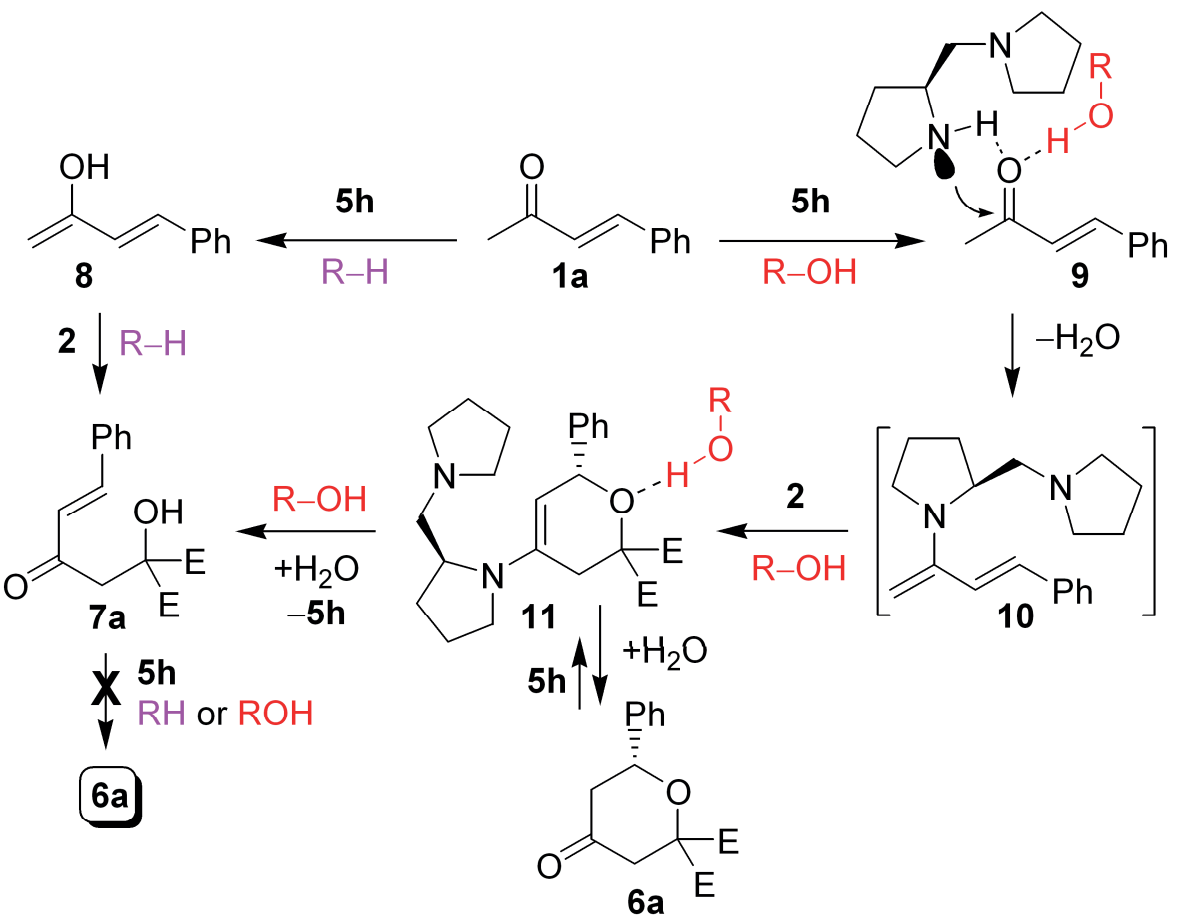

Scheme 2. Proposed reaction mechanism.

We further explored the scope of the (S)-1-(2-pyrrolidinylmethyl)pyrrolidine $\mathbf{5 h}$ catalyzed hetero-Diels-Alder reactions of various 4-substituted 3-buten-2-ones 1a-k with ketone $\mathbf{2}$ (Table 4). Even though the enantioselectivities were poor, chiral amine $\mathbf{5 h}$ was used as catalyst to study the chemoselective generation of chemically diverse tetrahydropyranones (chiral amine $\mathbf{5 h}$ is cheaper compared to racemic amine $\mathbf{5 h}$ ). ${ }^{28-30}$ Interestingly, amine $\mathbf{5 h}$ catalyzed the HDA reaction of arylidene acetones 1a-d with $\mathbf{2}$ to furnish the products $\mathbf{6}$ and $\mathbf{7}$ in a reasonable ratio as shown in Table 4, entries 1-4. The same reaction with alkylideneacetones $\mathbf{1 f}-\mathbf{j}$ furnished the chemoselective HDA products $\mathbf{6} \mathbf{f}-\mathbf{j}$ in very good yields (Table 4, entries 6-10); these compounds have direct application in total synthesis of bioactive natural products.

Table 4. Direct amine $\mathbf{5 h}$ catalyzed HDA reactions of different enones $\mathbf{1 a - k}$ with diethyl ketomalonate $\mathbf{2}^{a}$

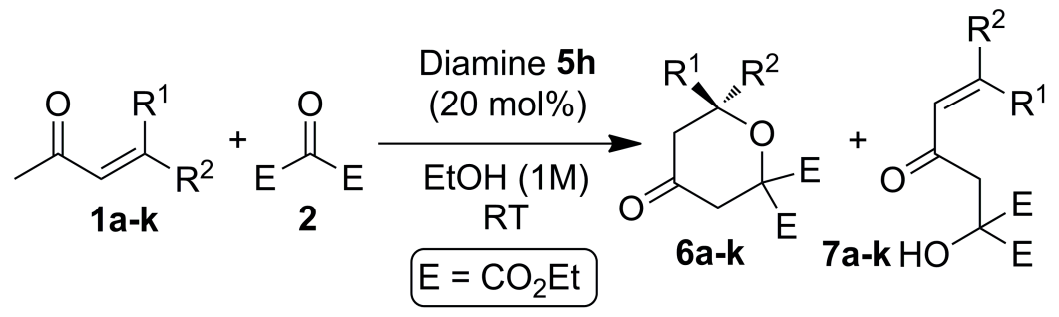


Table 4 (continued)

\begin{tabular}{ccccc}
\hline Entry & Enone & Time $(\mathrm{h})$ & Yield $(\%)^{b}$ & $\operatorname{Ratio}_{(\mathbf{6} / 7)^{c}}$ \\
\hline 1 & $\mathbf{1 a}$ & 12 & $\mathbf{6 a , 7 a ( 7 5 )}$ & $9: 1$ \\
2 & $\mathbf{1 b}$ & 68 & $\mathbf{6 b , 7 b}(65)$ & $1: 4$ \\
3 & $\mathbf{1 c}$ & 68 & $\mathbf{6 c}, \mathbf{7 c}(60)$ & $2.2: 1$ \\
4 & $\mathbf{1 d}$ & 78 & $\mathbf{6 d , 7 d}(65)$ & $1: 6.5$ \\
5 & $\mathbf{1 e}$ & 72 & $\mathbf{6 e , 7 e}(45)$ & $1: 2$ \\
6 & $\mathbf{1 f}$ & 78 & $\mathbf{6 f}(>99)$ & $99: 1$ \\
7 & $\mathbf{1 g}$ & 78 & $\mathbf{6 g}(>99)$ & $99: 1$ \\
8 & $\mathbf{1 h}$ & 78 & $\mathbf{6 h}(92)$ & $99: 1$ \\
9 & $\mathbf{1 i}$ & 78 & $\mathbf{6 i}(83)$ & $99: 1$ \\
10 & $\mathbf{1 j}$ & 120 & $\mathbf{6 j}(85)$ & $99: 1$ \\
11 & $\mathbf{1 k}$ & 72 & $\mathbf{6 k , 7 k}(54)$ & $1: 2$ \\
\hline
\end{tabular}

a See Supporting Information. ${ }^{b}$ Yield refers to the column-purified product. ${ }^{c}$ Ratio determined by ${ }^{1} \mathrm{H}$ NMR analysis.

We also evaluated the amine $\mathbf{5 h}$-catalyzed three-component Wittig/HDA reaction of phosphorane 3, aldehyde 4, and ketone $\mathbf{2}$ to furnish the tetrahydropyranes $\mathbf{6}$ in good yields as shown in Table 5. Aliphatic aldehyde $\mathbf{4 h}$ did not provide good yields (entry 2), but aromatic aldehydes 4a, 4l-p gave good yields of tandem Wittig/HDA products 6 and aldol products 7 (Table 5, entries 1, 3-7).

Table 5. Direct amine 5h catalyzed three-component Wittig/HDA reactions of $\mathbf{2}, \mathbf{3}$, and $\mathbf{4}^{a}$

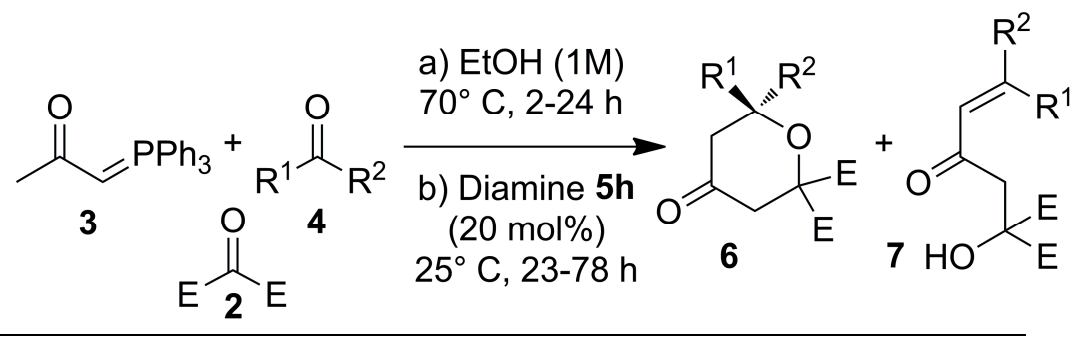

\begin{tabular}{ccccc}
\hline Entry & aldehyde & Time $(\mathrm{h})$ & ${\text { Yield }(\%)^{b}}^{b}$ & ${\text { Ratio }(\mathbf{6} / 7)^{c}}^{c}$ \\
\hline 1 & $\mathbf{4 a}$ & $2 / 23$ & $\mathbf{6 a , 7 a}(85)$ & $9: 1$ \\
2 & $\mathbf{4 h}$ & $24 / 75$ & $\mathbf{6 h}(20)$ & $99: 1$ \\
3 & $\mathbf{4 l}$ & $24 / 72$ & $\mathbf{6 l , 7 1}(97)$ & $2.4: 1$ \\
4 & $\mathbf{4 m}$ & $24 / 72$ & $\mathbf{6 m , 7 m}(91)$ & $1: 1$ \\
5 & $\mathbf{4 n}$ & $24 / 72$ & $\mathbf{6 n , 7 n}(80)$ & $3.3: 1$ \\
6 & $\mathbf{4 o}$ & $24 / 75$ & $\mathbf{6 o , 7 0}(52)$ & $1.6: 1$ \\
7 & $\mathbf{4 p}$ & $4 / 65$ & $\mathbf{6 p , 7} \mathbf{p}(60)$ & $2.7: 1$ \\
\hline
\end{tabular}

${ }^{a}$ See Supporting Information. ${ }^{b}$ Yield refers to the column-purified product. ${ }^{c}$ Ratio determined by ${ }^{1} \mathrm{H}$ NMR analysis 


\section{Conclusions}

In summary, we have developed methods for the asymmetric HDA and three-component Wittig/HDA reactions to produce substituted tetrahydropyranes 6 under amine-catalysis. The one-pot reaction proceeds in good yield with diamine $\mathbf{5 h}$ as the catalyst. Furthermore, we have demonstrated that the in situ generated 2-hydroxy-1,3-butadienes 8 via general acid/base-catalysis with chiral amines $\mathbf{5 h}-\mathbf{r}$ and amino acids 5a-g undergo aldol addition with 2 to yield 7. Further work is in progress to improve the ee of the reaction and utilize these novel HDA products in natural product synthesis.

\section{Experimental Section}

General. The ${ }^{1} \mathrm{H}$ NMR and ${ }^{13} \mathrm{C}$ NMR spectra were recorded at $400 \mathrm{MHz}$ and $100 \mathrm{MHz}$, respectively. The chemical shifts are reported in ppm downfield to TMS $(\delta=0)$ for ${ }^{1} \mathrm{H}$ NMR and relative to the central $\mathrm{CDCl}_{3}$ resonance $(\delta=77.0)$ for ${ }^{13} \mathrm{C}$ NMR. In the ${ }^{13} \mathrm{C}$ NMR spectra, the nature of the carbons $\left(\mathrm{C}, \mathrm{CH}, \mathrm{CH}_{2}\right.$ or $\left.\mathrm{CH}_{3}\right)$ was determined by recording the DEPT-135 experiment, and is given in parentheses. The coupling constants $J$ are given in Hz. Column chromatography was performed using Acme silica gel (particle size 0.063-0.200 mm). Highresolution mass spectra were recorded on micromass ESI-TOF MS. GCMS mass spectrometry was performed on Shimadzu GCMS-QP2010 mass spectrometer. Elemental analyses were recorded on a Thermo Finnigan Flash EA 1112 analyzer. LCMS mass spectra were recorded on either $\mathrm{VG} 7070 \mathrm{H}$ mass spectrometer using EI technique or ShimadzuLCMS-2010A mass spectrometer. IR spectra were recorded on JASCO FT/IR-5300. For thinlayer chromatography (TLC), silica gel plates Merck 60 F254 were used and compounds were visualized by irradiation with UV light and/or by treatment with a solution of $p$ anisaldehyde $(23 \mathrm{~mL})$, conc. $\mathrm{H}_{2} \mathrm{SO}_{4}(35 \mathrm{~mL})$, acetic acid $(10 \mathrm{~mL})$, and ethanol $(900 \mathrm{~mL})$ followed by heating.

Materials. All solvents and commercially available chemicals were used as received.

\section{General experimental procedures for the organocatalytic reactions}

Chiral amine or amino acid-catalyzed asymmetric hetero-Diels-Alder reactions

In an ordinary glass vial equipped with a magnetic stirring bar, to $1.0 \mathrm{mmol}$ of the enone 1 and $1.0 \mathrm{~mL}$ of solvent, catalyst amine $5(20 \mathrm{~mol} \%)$ was added and the reaction mixture was stirred at ambient temperature for 5 minutes. To the reaction mixture $0.5 \mathrm{mmol}$ of diethyl ketomalonate 2 was added and stirred at ambient temperature for the time indicated in Tables $1,2,3$ and 4. The crude reaction mixture was directly loaded onto a silica gel column with or without aqueous work-up and pure hetero-Diels-Alder 6 and aldol 7 products were obtained by flash column chromatography (silica gel; hexane/ethyl acetate mixture).

\section{(S)-1-(2-Pyrrolidinylmethyl)pyrrolidine}

\section{reactions in one-pot}

In an ordinary glass vial equipped with a magnetic stirring bar, to $0.6 \mathrm{mmol}$ of the phosphorane 3 and $1.0 \mathrm{~mL}$ of EtOH, $0.6 \mathrm{mmol}$ of the aldehyde 4 was added and the reaction 
mixture was stirred at $70^{\circ} \mathrm{C}$ for the time indicated in Table 5. To the reaction mixture catalyst amine $\mathbf{5 h}$ (20 mol-\%) was added and the reaction mixture was stirred at ambient temperature for 5 minutes. Then $0.3 \mathrm{mmol}$ of diethylketomalonate 2 was added and stirred at ambient temperature for the time indicated in Table 5. The crude reaction mixture was directly loaded on silica gel column with or without aqueous work-up and pure tandem Wittig/hetero-DielsAlder 6 and Wittig/aldol 7 products were obtained by flash column chromatography (silica gel, mixture of hexane/ethyl acetate). All new compounds were characterized on the basis of IR, ${ }^{1} \mathrm{H}$ and ${ }^{13} \mathrm{C}$ NMR and analytical data (see Supporting Information).

(6R)-4-Oxo-6-phenyltetrahydropyran-2,2-dicarboxylic acid diethyl ester (6a). Purified by

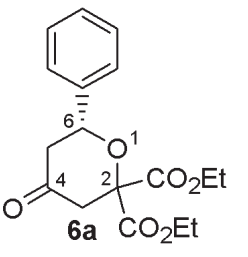
column chromatography using EtOAc/hexane and isolated as oil. The ee was determined by chiral-phase HPLC using a Daicel Chiralcell OD-H column (hexane $/ i-\mathrm{PrOH}=85: 15$, flow rate $1.0 \mathrm{~mL} / \mathrm{min}, \lambda=220 \mathrm{~nm}$ ), $t_{\mathrm{R}}=10.71 \mathrm{~min}$ (minor), $t_{\mathrm{R}}=13.02$ min (major); IR (neat): $v_{\max } 2984,1743(\mathrm{C}=\mathrm{O}, \mathrm{O}-\mathrm{C}=\mathrm{O})$, $1608,1452,1369,1224,1070,858,760,700 \mathrm{~cm}^{-1} ;{ }^{1} \mathrm{H} \mathrm{NMR}\left(\mathrm{CDCl}_{3}\right) \delta 7.40-$ $7.23(5 \mathrm{H}, \mathrm{m}, \mathrm{Ph}-H), 4.90(1 \mathrm{H}, \mathrm{dd}, J=9.2,5.2 \mathrm{~Hz}), 4.27(4 \mathrm{H}$, br q, $J=7.2 \mathrm{~Hz}, 2 \times$ $\left.\mathrm{OCH}_{2} \mathrm{CH}_{3}\right), 3.15(1 \mathrm{H}, \mathrm{d}, J=15.6 \mathrm{~Hz}), 2.80(1 \mathrm{H}, \mathrm{d}, J=15.6 \mathrm{~Hz}), 2.63(2 \mathrm{H}, \mathrm{m}), 1.264(3 \mathrm{H}, \mathrm{t}, J$ $=7.2 \mathrm{~Hz}), 1.259(3 \mathrm{H}, \mathrm{t}, J=7.2 \mathrm{~Hz})\left[2 \times \mathrm{OCH}_{2} \mathrm{CH}_{3}\right] ;{ }^{13} \mathrm{C} \mathrm{NMR}\left(\mathrm{CDCl}_{3}, \mathrm{DEPT}\right) \delta 202.2(\mathrm{C}$, $\mathrm{C}=\mathrm{O}), 167.5(\mathrm{C}, \mathrm{O}=\mathrm{C}-\mathrm{O}), 167.0(\mathrm{C}, \mathrm{O}=\mathrm{C}-\mathrm{O}), 139.3(\mathrm{C}), 128.6(2 \times \mathrm{CH}), 128.4(\mathrm{CH}), 125.9$ $(2 \times \mathrm{CH}), 82.2(\mathrm{C}, \mathrm{C}-2), 75.6(\mathrm{CH}, \mathrm{C}-6), 62.6\left(2 \times \mathrm{CH}_{2}, \mathrm{OCH}_{2} \mathrm{CH}_{3}\right), 47.9\left(\mathrm{CH}_{2}\right), 44.2\left(\mathrm{CH}_{2}\right)$, $13.96\left(\mathrm{CH}_{3}\right), 13.90\left(\mathrm{CH}_{3}\right)\left[2 \times \mathrm{OCH}_{2} \mathrm{CH}_{3}\right]$; HRMS (ESI-TOF): $m / z 321.1331\left(\mathrm{M}+\mathrm{H}^{+}\right)$, calcd for $\mathrm{C}_{17} \mathrm{H}_{20} \mathrm{O}_{6} \mathrm{H}^{+} 321.1333$.

2-Hydroxy-2-(2-oxo-4-phenylbut-3-enyl)malonic acid diethyl ester (7a). Purified by

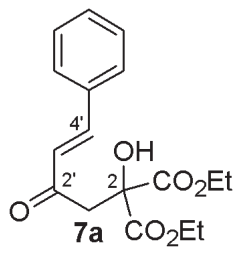
column chromatography using EtOAc/hexane and isolated as oil. IR (neat): $v_{\max } 3473(\mathrm{O}-\mathrm{H}), 2984,1739(\mathrm{C}=\mathrm{O}, \mathrm{O}-\mathrm{C}=\mathrm{O}), 1693,1665(\mathrm{C}=\mathrm{C}), 1611$, $1281,1231 \mathrm{~cm}^{-1} ;{ }^{1} \mathrm{H}$ NMR $\left(\mathrm{CDCl}_{3}\right) \delta 7.56(1 \mathrm{H}, \mathrm{d}, J=16.4 \mathrm{~Hz}$, olefinic- $\beta$ $H), 7.50(1 \mathrm{H}, \mathrm{m}), 7.37(4 \mathrm{H}, \mathrm{m}), 6.70(1 \mathrm{H}, \mathrm{d}, J=16.4 \mathrm{~Hz}$, olefinic- $\alpha-H)$, $4.27\left(4 \mathrm{H}, \mathrm{q}, J=7.2 \mathrm{~Hz}, 2 \times \mathrm{OCH}_{2} \mathrm{CH}_{3}\right), 3.80(1 \mathrm{H}, \mathrm{dd}, J=3.6,2.0 \mathrm{~Hz}, \mathrm{O}-$ $H), 3.51\left(2 \mathrm{H}, \mathrm{s}, \mathrm{CH}_{2}\right), 1.26\left(6 \mathrm{H}, \mathrm{t}, J=7.2 \mathrm{~Hz}, 2 \times \mathrm{OCH}_{2} \mathrm{CH}_{3}\right) ;{ }^{13} \mathrm{C} \mathrm{NMR}\left(\mathrm{CDCl}_{3}, \mathrm{DEPT}\right) \delta$ $196.2(\mathrm{C}, \mathrm{C}=\mathrm{O}), 169.5(2 \times \mathrm{C}, \mathrm{O}=\mathrm{C}-\mathrm{O}), 144.1(\mathrm{CH}), 134.0(\mathrm{C}), 130.8(\mathrm{CH}), 128.9(2 \times \mathrm{CH})$, $128.4(2 \times \mathrm{CH}), 125.7(\mathrm{CH}), 76.8(\mathrm{C}, \mathrm{C}-\mathrm{OH}), 62.70\left(2 \times \mathrm{CH}_{2}, \mathrm{OCH}_{2} \mathrm{CH}_{3}\right), 44.9\left(\mathrm{CH}_{2}\right), 13.9$ $\left(2 \times \mathrm{CH}_{3}, \mathrm{OCH}_{2} \mathrm{CH}_{3}\right)$; HRMS (ESI-TOF): $\mathrm{m} / z 321.1330\left(\mathrm{M}+\mathrm{H}^{+}\right)$, calcd for $\mathrm{C}_{17} \mathrm{H}_{20} \mathrm{O}_{6} \mathrm{H}^{+}$ 321.1333 .

2-Hydroxy-2-[4-(4-hydroxyphenyl)-2-oxobut-3-enyl]malonic acid diethyl ester (7b).

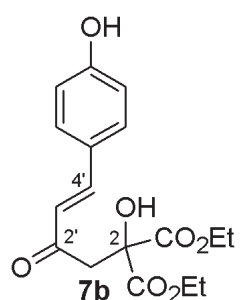

Purified by column chromatography using EtOAc/hexane and isolated as oil. IR (neat): $v_{\max } 3420(\mathrm{O}-\mathrm{H}), 2986,1747(\mathrm{C}=\mathrm{O}, \mathrm{O}-\mathrm{C}=\mathrm{O}), 1647,1601$, 1516, 1445, 1369, 1234, 856, 820, $737 \mathrm{~cm}^{-1} ;{ }^{1} \mathrm{H} \mathrm{NMR}\left(\mathrm{CDCl}_{3}\right) \delta 7.50(1 \mathrm{H}$, $\mathrm{d}, J=16.4 \mathrm{~Hz}$, olefinic- $\beta-H), 7.39(2 \mathrm{H}, \mathrm{d}, J=8.4 \mathrm{~Hz}, \mathrm{Ph}-H), 7.23(1 \mathrm{H}, \mathrm{br}$, Ar-OH), $6.87(2 \mathrm{H}, \mathrm{d}, J=8.4 \mathrm{~Hz}, \mathrm{Ph}-H), 6.54(1 \mathrm{H}, \mathrm{d}, J=16.0 \mathrm{~Hz}$, olefinic$\alpha-H), 4.51\left(1 \mathrm{H}\right.$, br s, O-H), $4.30\left(4 \mathrm{H}\right.$, br q $\left., J=6.8 \mathrm{~Hz}, 2 \times \mathrm{OCH}_{2} \mathrm{CH}_{3}\right)$,

$3.51\left(2 \mathrm{H}, \mathrm{s}, \mathrm{CH}_{2}\right), 1.29\left(6 \mathrm{H}\right.$, br t, $\left.J=7.2 \mathrm{~Hz}, 2 \times \mathrm{OCH}_{2} \mathrm{CH}_{3}\right) ;{ }^{13} \mathrm{C} \mathrm{NMR}\left(\mathrm{CDCl}_{3}, \mathrm{DEPT}\right) \delta$ $196.7(\mathrm{C}, \mathrm{C}=\mathrm{O}), 169.6(2 \times \mathrm{C}, \mathrm{O}=\mathrm{C}-\mathrm{O}), 159.1(\mathrm{C}, \mathrm{C}-\mathrm{OH}), 144.7(\mathrm{CH}$, olefinic- $\beta-\mathrm{CH}), 130.5$ $(2 \times \mathrm{CH}), 126.1(\mathrm{C}), 122.9(\mathrm{CH}$, olefinic- $\alpha-\mathrm{CH}), 116.1(2 \times \mathrm{CH}), 77.1(\mathrm{C}, \mathrm{C}-2), 62.8(2 \times$ 
$\left.\mathrm{CH}_{2}, \mathrm{OCH}_{2} \mathrm{CH}_{3}\right), 44.7\left(\mathrm{CH}_{2}\right), 13.9\left(2 \times \mathrm{CH}_{3}, \mathrm{OCH}_{2} \mathrm{CH}_{3}\right)$; HRMS (ESI-TOF): $\mathrm{m} / z 359.1115$ $\left(\mathrm{M}+\mathrm{Na}^{+}\right)$, calcd for $\mathrm{C}_{17} \mathrm{H}_{20} \mathrm{O}_{7} \mathrm{Na}^{+}$359.1107; LRMS: $m / z 337.10(\mathrm{M}+1)$, calcd for $\mathrm{C}_{17} \mathrm{H}_{20} \mathrm{O}_{7}$ 336.1209 .

6-(2,6-Dichlorophenyl)-4-oxotetrahydropyran-2,2-dicarboxylic acid diethyl ester (6c).

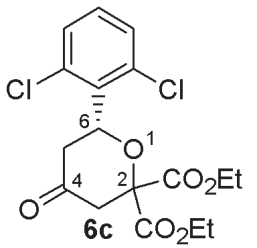

Purified by column chromatography using EtOAc/hexane and isolated as oil. IR (neat): $v_{\max } 2984,2926,1699(\mathrm{C}=\mathrm{O}, \mathrm{O}-\mathrm{C}=\mathrm{O}), 1558,1458,858,771$, $636 \mathrm{~cm}^{-1} ;{ }^{1} \mathrm{H}$ NMR $\left(\mathrm{CDCl}_{3}\right) \delta 7.38-7.30(3 \mathrm{H}, \mathrm{m}, \mathrm{Ph}-H), 5.78(1 \mathrm{H}, \mathrm{dd}, J=$ $\left.12.0,4.0 \mathrm{~Hz}, \mathrm{C}_{6}-\mathrm{H}\right), 4.29\left(4 \mathrm{H}, \mathrm{q}, J=7.2 \mathrm{~Hz}, 2 \times \mathrm{OCH}_{2} \mathrm{CH}_{3}\right), 3.33(1 \mathrm{H}$, dd, $J=15.6,12.0 \mathrm{~Hz}), 3.17(1 \mathrm{H}, \mathrm{br} \mathrm{d}, J=16.4 \mathrm{~Hz}), 2.89(1 \mathrm{H}, \mathrm{d}, J=15.2 \mathrm{~Hz})$, $2.50(1 \mathrm{H}, \mathrm{ddd}, J=15.6,10.0,2.0 \mathrm{~Hz}), 1.29\left(6 \mathrm{H}, \mathrm{t}, J=7.2 \mathrm{~Hz}, 2 \times \mathrm{OCH}_{2} \mathrm{CH}_{3}\right) ;{ }^{13} \mathrm{C} \mathrm{NMR}$ $\left(\mathrm{CDCl}_{3}, \mathrm{DEPT}\right) \delta 201.6(\mathrm{C}, \mathrm{C}=\mathrm{O}), 169.4(\mathrm{C}, \mathrm{O}=\mathrm{C}-\mathrm{O}), 166.5(\mathrm{C}, \mathrm{O}=\mathrm{C}-\mathrm{O}), 135.2(\mathrm{C}, \mathrm{C}-\mathrm{Cl})$, 135.1 (C, C-Cl), 132.7 (C, C-7), $130.1(2 \times \mathrm{CH}, \mathrm{Ph}-\mathrm{CH}), 128.8$ (CH, Ph- $\mathrm{CH}), 82.3$ (C, C-2), $72.4(\mathrm{CH}, \mathrm{C}-6), 62.8\left(\mathrm{CH}_{2}\right), 62.6\left(\mathrm{CH}_{2}\right)\left[2 \times \mathrm{OCH}_{2} \mathrm{CH}_{3}\right] ; 44.3\left(\mathrm{CH}_{2}\right), 42.4\left(\mathrm{CH}_{2}\right), 14.0\left(\mathrm{CH}_{3}\right.$, $\left.\mathrm{OCH}_{2} \mathrm{CH}_{3}\right), 13.9\left(\mathrm{CH}_{3}, \mathrm{OCH}_{2} \mathrm{CH}_{3}\right)$; LRMS: $m / z 389.10(\mathrm{M}+1)$, calcd for $\mathrm{C}_{17} \mathrm{H}_{18} \mathrm{Cl}_{2} \mathrm{O}_{6}$ 388.0480; HRMS (ESI-TOF): $m / z 411.0389\left(\mathrm{M}+\mathrm{Na}^{+}\right.$), calcd for $\mathrm{C}_{17} \mathrm{H}_{18} \mathrm{Cl}_{2} \mathrm{O}_{6} \mathrm{Na}^{+} 411.0378$.

2-[4-(2,6-Dichlorophenyl)-2-oxobut-3-enyl]-2-hydroxymalonic acid diethyl ester (7c).

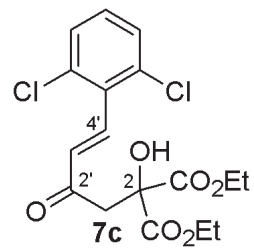
Purified by column chromatography using EtOAc/hexane and isolated as oil. IR (neat): $v_{\max } 3503(\mathrm{O}-\mathrm{H}), 2984,2926,1699(\mathrm{C}=\mathrm{O}, \mathrm{O}-\mathrm{C}=\mathrm{O}), 1558$, $1458,858,771,636 \mathrm{~cm}^{-1} ;{ }^{1} \mathrm{H} \mathrm{NMR}\left(\mathrm{CDCl}_{3}\right) \delta 7.68(1 \mathrm{H}, \mathrm{d}, J=16.4 \mathrm{~Hz}$, olefinic- $\beta-H), 7.22-7.17(3 \mathrm{H}, \mathrm{m}, \mathrm{Ph}-H), 6.87(1 \mathrm{H}, \mathrm{d}, J=16.4 \mathrm{~Hz}$, olefinic$\alpha-H), 4.30\left(4 \mathrm{H}, \mathrm{q}, J=7.2 \mathrm{~Hz}, 2 \times \mathrm{OCH}_{2} \mathrm{CH}_{3}\right), 3.56\left(2 \mathrm{H}, \mathrm{s}, \mathrm{CH}_{2}\right), 1.29(6 \mathrm{H}$, t, $\left.J=7.2 \mathrm{~Hz}, 2 \times \mathrm{OCH}_{2} \mathrm{CH}_{3}\right) ;{ }^{13} \mathrm{C} \mathrm{NMR}\left(\mathrm{CDCl}_{3}\right.$, DEPT $) \delta 195.8(\mathrm{C}, \mathrm{C}=\mathrm{O}), 167.6(2 \times \mathrm{C}$, $\mathrm{O}=\mathrm{C}-\mathrm{O}), 137.2(\mathrm{CH}$, olefinic- $\beta-\mathrm{CH}), 133.4(\mathrm{CH}$, olefinic- $\alpha-\mathrm{CH}), 131.6(2 \times \mathrm{C}), 129.6(2 \times$ $\mathrm{CH}), 127.8(\mathrm{CH}), 77.2(\mathrm{C}, \mathrm{C}-\mathrm{OH}), 62.8\left(2 \times \mathrm{CH}_{2}, \mathrm{OCH}_{2} \mathrm{CH}_{3}\right), 45.5\left(\mathrm{CH}_{2}\right), 13.9\left(2 \times \mathrm{CH}_{3}\right.$, $\mathrm{OCH}_{2} \mathrm{CH}_{3}$ ); LRMS: $m / z 389.10(\mathrm{M}+1)$, calcd for $\mathrm{C}_{17} \mathrm{H}_{18} \mathrm{Cl}_{2} \mathrm{O}_{6} 388.0480$; HRMS (ESI-TOF): $m / z 411.0389\left(\mathrm{M}+\mathrm{Na}^{+}\right)$, calcd for $\mathrm{C}_{17} \mathrm{H}_{18} \mathrm{Cl}_{2} \mathrm{O}_{6} \mathrm{Na}^{+} 411.0378$.

4-Oxo-6-styryltetrahydropyran-2,2-dicarboxylic acid diethyl ester (6d). Purified by

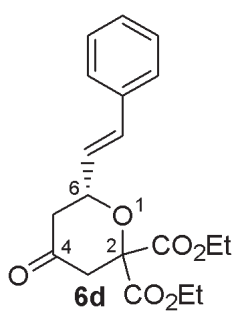
column chromatography using EtOAc/hexane and isolated as oil. IR (neat): $v_{\max }$ 2982, $1742(\mathrm{C}=\mathrm{O}, \mathrm{O}-\mathrm{C}=\mathrm{O}), 1618(\mathrm{C}=\mathrm{C}), 1450,1365,1282,1232$, 1014, 858, 750, $694 \mathrm{~cm}^{-1} ;{ }^{1} \mathrm{H}$ NMR $\left(\mathrm{CDCl}_{3}\right) \delta$ 7.40-7.26 (5H, m, Ph- $\left.H\right)$, $6.65(1 \mathrm{H}, \mathrm{d}, J=16.0 \mathrm{~Hz}), 6.29(1 \mathrm{H}, \mathrm{dd}, J=16.0,6.4 \mathrm{~Hz}), 4.57(1 \mathrm{H}, \mathrm{dd}, J=$ $\left.14.4,6.4 \mathrm{~Hz}, \mathrm{C}_{6}-\mathrm{H}\right), 4.30\left(4 \mathrm{H}, \mathrm{q}, J=7.2 \mathrm{~Hz}, 2 \times \mathrm{OCH}_{2} \mathrm{CH}_{3}\right), 3.12(1 \mathrm{H}, \mathrm{d}, J$ $=15.6 \mathrm{~Hz}), 2.77(1 \mathrm{H}, \mathrm{d}, J=15.6 \mathrm{~Hz}), 2.57(2 \mathrm{H}$, br d, $J=8.0 \mathrm{~Hz}), 1.30(6 \mathrm{H}$,

$\left.\mathrm{t}, J=7.2 \mathrm{~Hz}, 2 \times \mathrm{OCH}_{2} \mathrm{CH}_{3}\right) ;{ }^{13} \mathrm{C} \mathrm{NMR}\left(\mathrm{CDCl}_{3}\right.$, DEPT) $\delta 202.0(\mathrm{C}, \mathrm{C}=\mathrm{O})$, $167.5(\mathrm{C}, \mathrm{O}=\mathrm{C}-\mathrm{O}), 167.0(\mathrm{C}, \mathrm{O}=\mathrm{C}-\mathrm{O}), 135.7(\mathrm{C}), 132.8(\mathrm{CH}), 128.6(2 \times \mathrm{CH}, \mathrm{Ph}-\mathrm{CH}), 128.2$ $(\mathrm{CH}), 126.8(\mathrm{CH}), 126.7(2 \times \mathrm{CH}, \mathrm{Ph}-\mathrm{CH}), 82.0(\mathrm{C}, \mathrm{C}-2), 74.7(\mathrm{CH}, \mathrm{C}-6), 62.7\left(2 \times \mathrm{CH}_{2}\right.$, $\left.\mathrm{OCH}_{2} \mathrm{CH}_{3}\right), 46.2\left(\mathrm{CH}_{2}\right), 44.3\left(\mathrm{CH}_{2}\right), 13.9\left(2 \times \mathrm{CH}_{3}, \mathrm{OCH}_{2} \mathrm{CH}_{3}\right) ; \mathrm{HRMS}$ (ESI-TOF): $\mathrm{m} / z$ $369.1328\left(\mathrm{M}+\mathrm{Na}^{+}\right)$, calcd for $\mathrm{C}_{19} \mathrm{H}_{22} \mathrm{O}_{6} \mathrm{Na}^{+} 369.1314$.

2-Hydroxy-2-(2-oxo-6-phenylhexa-3,5-dienyl)malonic acid diethyl ester (7d). Purified by

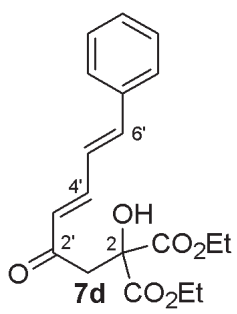

column chromatography using EtOAc/hexane and isolated as oil. IR (neat): $v_{\max } 3474(\mathrm{O}-\mathrm{H}), 2982,1742(\mathrm{C}=\mathrm{O}, \mathrm{O}-\mathrm{C}=\mathrm{O}), 1660(\mathrm{O}=\mathrm{C}-\mathrm{C}=\mathrm{C}), 1618$ $(\mathrm{C}=\mathrm{C}), 1450,1365,1282,1232,1014,858,750,694 \mathrm{~cm}^{-1} ;{ }^{1} \mathrm{H} \mathrm{NMR}\left(\mathrm{CDCl}_{3}\right)$ $\delta 7.48(2 \mathrm{H}, \mathrm{d}, J=6.8 \mathrm{~Hz}, \mathrm{Ph}-H), 7.41-7.32$ (4H, m, Ph- $H$, olefinic- $H), 6.99$ 
$\left(1 \mathrm{H}, \mathrm{d}, J=15.2 \mathrm{~Hz}, \mathrm{C}_{6^{\prime}}-H\right), 6.88(1 \mathrm{H}, \mathrm{dd}, J=15.6,10.8 \mathrm{~Hz}$, olefinic- $H), 6.27(1 \mathrm{H}, \mathrm{d}, J=15.6$ $\mathrm{Hz}$, olefinic- $H), 4.29\left(4 \mathrm{H}, \mathrm{q}, J=7.2 \mathrm{~Hz}, 2 \times \mathrm{OCH}_{2} \mathrm{CH}_{3}\right), 3.47\left(2 \mathrm{H}, \mathrm{s}, \mathrm{CH}_{2}\right), 1.29(6 \mathrm{H}, \mathrm{t}, J=$ $\left.7.2 \mathrm{~Hz}, 2 \times \mathrm{OCH}_{2} \mathrm{CH}_{3}\right) ;{ }^{13} \mathrm{C} \mathrm{NMR}\left(\mathrm{CDCl}_{3}\right.$, DEPT $) \delta 196.2(\mathrm{C}, \mathrm{C}=\mathrm{O}), 169.5(2 \times \mathrm{C}, \mathrm{O}=\mathrm{C}-\mathrm{O})$, 144.1 (CH, C-6'), 142.3 (CH, C-5'), 135.7 (C, C-7'), 129.3 (CH, C-4'), 128.9 (CH, C-3'), $128.8(2 \times \mathrm{CH}, \mathrm{Ph}-\mathrm{CH}), 127.3(2 \times \mathrm{CH}, \mathrm{Ph}-\mathrm{CH}), 126.2(\mathrm{CH}, \mathrm{Ph}-\mathrm{CH}), 76.8(\mathrm{C}, \mathrm{C}-\mathrm{OH}), 62.6$ $\left(2 \times \mathrm{CH}_{2}, \mathrm{OCH}_{2} \mathrm{CH}_{3}\right), 44.7\left(\mathrm{CH}_{2}\right), 13.8\left(2 \times \mathrm{CH}_{3}, \mathrm{OCH}_{2} \mathrm{CH}_{3}\right) ; \mathrm{HRMS}$ (ESI-TOF): $\mathrm{m} / z$ $369.1328\left(\mathrm{M}+\mathrm{Na}^{+}\right)$, calcd for $\mathrm{C}_{19} \mathrm{H}_{22} \mathrm{O}_{6} \mathrm{Na}^{+} 369.1314$.

4-Oxo-6-propenyltetrahydropyran-2,2-dicarboxylic acid diethyl ester (6e). Purified by

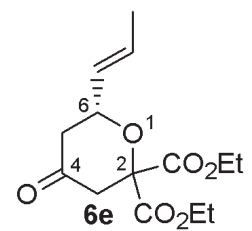

column chromatography using EtOAc/hexane and isolated as oil. IR (neat): $v_{\max } 2984,2932,1744(\mathrm{C}=\mathrm{O}, \mathrm{O}-\mathrm{C}=\mathrm{O}), 1634(\mathrm{C}=\mathrm{C}), 1595,1447,1369$, $1287,1233,1136,1097,1020,860,783 \mathrm{~cm}^{-1} ;{ }^{1} \mathrm{H} \mathrm{NMR}\left(\mathrm{CDCl}_{3}\right) \delta 5.82-5.75$ $(1 \mathrm{H}, \mathrm{m}), 5.60(1 \mathrm{H}$, br dd, $J=15.6,6.8 \mathrm{~Hz})$ [olefinic- $H$ ]; 4.53-4.40 $(1 \mathrm{H}, \mathrm{m}$, $\left.\mathrm{C}_{6}-\mathrm{H}\right), 4.31\left(4 \mathrm{H}, \mathrm{m}, 2 \times \mathrm{OCH}_{2} \mathrm{CH}_{3}\right), 3.08(1 \mathrm{H}, \mathrm{d}, J=15.2 \mathrm{~Hz}), 2.69(1 \mathrm{H}, \mathrm{d}, J$ $=15.2 \mathrm{~Hz}), 2.45(2 \mathrm{H}, \mathrm{d}, J=7.2 \mathrm{~Hz}), 1.72(3 \mathrm{H}, \mathrm{d}, J=6.4 \mathrm{~Hz}, \mathrm{CH}=\mathrm{CHCH}), 1.30(6 \mathrm{H}, \mathrm{t}, J=$ $\left.7.2 \mathrm{~Hz}, 2 \times \mathrm{OCH}_{2} \mathrm{CH}_{3}\right) ;{ }^{13} \mathrm{C} \mathrm{NMR}\left(\mathrm{CDCl}_{3}\right.$, DEPT) $\delta 202.4(\mathrm{C}, \mathrm{C}=\mathrm{O}), 168.3(2 \times \mathrm{C}, \mathrm{O}=\mathrm{C}-\mathrm{O})$, $130.2(\mathrm{CH}), 129.1(\mathrm{CH})\left[\mathrm{CH}=\mathrm{CHCH}_{3}\right] ; 90.0(\mathrm{C}, \mathrm{C}-2), 74.8(\mathrm{CH}, \mathrm{C}-6), 62.6\left(2 \times \mathrm{CH}_{2}\right.$, $\left.\mathrm{OCH}_{2} \mathrm{CH}_{3}\right), 46.2\left(\mathrm{CH}_{2}\right), 44.1\left(\mathrm{CH}_{2}\right), 17.7\left(\mathrm{CH}_{3}, \mathrm{CH}=\mathrm{CHCH}_{3}\right), 13.84\left(2 \times \mathrm{CH}_{3}, \mathrm{OCH}_{2} \mathrm{CH}_{3}\right)$; LRMS: $m / z 285.10(\mathrm{M}+1)$, calcd for $\mathrm{C}_{14} \mathrm{H}_{20} \mathrm{O}_{6} 284.1260$.

2-Hydroxy-2-(2-oxohepta-3,5-dienyl)malonic acid diethyl ester (7e). Purified by column

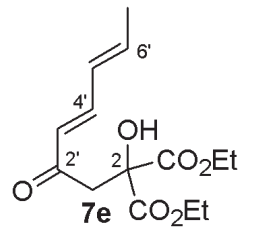

chromatography using EtOAc/hexane and isolated as oil. IR (neat): $v_{\max }$ $3460(\mathrm{O}-\mathrm{H}), 2984,2932,1744(\mathrm{C}=\mathrm{O}, \mathrm{O}-\mathrm{C}=\mathrm{O}), 1634(\mathrm{C}=\mathrm{C}), 1595,1447$, $1369,1287,1233,1136,1097,1020,860,783 \mathrm{~cm}^{-1} ;{ }^{1} \mathrm{H}$ NMR $\left(\mathrm{CDCl}_{3}\right) \delta$ $7.18(1 \mathrm{H}, \mathrm{dd}, J=15.2,9.2 \mathrm{~Hz}), 6.22(2 \mathrm{H}, \mathrm{m}), 6.05(1 \mathrm{H}, \mathrm{d}, J=15.6 \mathrm{~Hz}$, olefinic-H), $4.31\left(4 \mathrm{H}, \mathrm{m}, 2 \times \mathrm{OCH}_{2} \mathrm{CH}_{3}\right), 3.41\left(2 \mathrm{H}, \mathrm{s}, \mathrm{CH}_{2}\right), 1.88(3 \mathrm{H}, \mathrm{d}, J$ $\left.=6.4 \mathrm{~Hz}, \mathrm{CH}=\mathrm{CHCH}_{3}\right), 1.31\left(6 \mathrm{H}, \mathrm{t}, J=7.2 \mathrm{~Hz}, 2 \times \mathrm{OCH}_{2} \mathrm{CH}_{3}\right) ;{ }^{13} \mathrm{C} \mathrm{NMR}\left(\mathrm{CDCl}_{3}, \mathrm{DEPT}\right) \delta$ $196.7(\mathrm{C}, \mathrm{C}=\mathrm{O}), 169.5(2 \times \mathrm{C}, \mathrm{O}=\mathrm{C}-\mathrm{O}), 144.6(\mathrm{CH}), 141.7(\mathrm{CH}), 130.1(\mathrm{CH}), 127.1(\mathrm{CH})$, $76.9(\mathrm{C}, \mathrm{C}-\mathrm{OH}), 63.4\left(2 \times \mathrm{CH}_{2}, \mathrm{OCH}_{2} \mathrm{CH}_{3}\right), 44.5\left(\mathrm{CH}_{2}\right), 18.8\left(\mathrm{CH}_{3}, \mathrm{CH}=\mathrm{CHCH}_{3}\right), 13.9(2 \times$ $\left.\mathrm{CH}_{3}, \mathrm{OCH}_{2} \mathrm{CH}_{3}\right)$; LRMS: $m / z 285.10(\mathrm{M}+1)$, calcd for $\mathrm{C}_{14} \mathrm{H}_{20} \mathrm{O}_{6} 284.1260$.

6-Methyl-4-oxotetrahydropyran-2,2-dicarboxylic acid diethyl ester (6f). Purified by

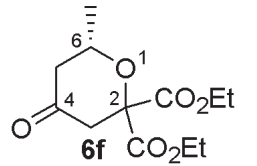

column chromatography using EtOAc/hexane and isolated as oil. IR (neat): $v_{\max } 2982,2936,1744(\mathrm{C}=\mathrm{O}, \mathrm{O}-\mathrm{C}=\mathrm{O}), 1628,1449,1385,1277,856 \mathrm{~cm}^{-1}$; ${ }^{1} \mathrm{H} \mathrm{NMR}\left(\mathrm{CDCl}_{3}\right) \delta 4.33-4.26\left(4 \mathrm{H}\right.$, br q, $\left.J=6.8 \mathrm{~Hz}, 2 \times \mathrm{OCH}_{2} \mathrm{CH}_{3}\right), 3.99$ $\left(1 \mathrm{H}, \mathrm{m}, \mathrm{C}_{6}-\mathrm{H}\right), 3.06(1 \mathrm{H}, \mathrm{d}, J=16 \mathrm{~Hz}), 2.64(1 \mathrm{H}, \mathrm{d}, J=15.2 \mathrm{~Hz}), 2.43(1 \mathrm{H}$, br d, $J=15.6 \mathrm{~Hz}), 2.32(1 \mathrm{H}, \mathrm{dd}, J=15.2,11.6 \mathrm{~Hz}), 1.42\left(3 \mathrm{H}, \mathrm{d}, J=6 \mathrm{~Hz}, \mathrm{C}_{6}-\mathrm{CH}_{3}\right), 1.30-1.27$ $\left(6 \mathrm{H}\right.$, br t, $\left.J=6.8 \mathrm{~Hz}, 2 \times \mathrm{OCH}_{2} \mathrm{CH}_{3}\right) ;{ }^{13} \mathrm{C} \mathrm{NMR}\left(\mathrm{CDCl}_{3}, \mathrm{DEPT}\right) \delta 202.5(\mathrm{C}, \mathrm{C}=\mathrm{O}), 167.5(\mathrm{C}$, $\mathrm{O}-\mathrm{C}=\mathrm{O}), 167.0(\mathrm{C}, \mathrm{O}-\mathrm{C}=\mathrm{O}), 82.0(\mathrm{C}, \mathrm{C}-2), 70.3(\mathrm{CH}, \mathrm{C}-6), 62.4\left(\mathrm{CH}_{2}, \mathrm{OCH}_{2} \mathrm{CH}_{3}\right), 62.3$ $\left(\mathrm{CH}_{2}, \mathrm{OCH}_{2} \mathrm{CH}_{3}\right), 47.6\left(\mathrm{CH}_{2}\right), 43.9\left(\mathrm{CH}_{2}\right), 21.7\left(\mathrm{CH}_{3}\right), 13.8\left(\mathrm{CH}_{3}, \mathrm{OCH}_{2} \mathrm{CH}_{3}\right), 13.9\left(\mathrm{CH}_{3}\right.$, $\mathrm{OCH}_{2} \mathrm{CH}_{3}$ ); HRMS (ESI-TOF): $m / z 281.0988\left(\mathrm{M}+\mathrm{Na}^{+}\right.$), calcd for $\mathrm{C}_{12} \mathrm{H}_{18} \mathrm{O}_{6} \mathrm{Na}^{+} 281.1001$.

4-Oxo-6-propyltetrahydropyran-2,2-dicarboxylic acid diethyl ester (6g). Purified by

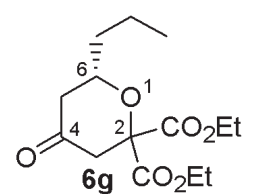
column chromatography using EtOAc/hexane and isolated as oil. IR (neat): $\nu_{\max } 2963,2880,1746(\mathrm{C}=\mathrm{O}, \mathrm{O}-\mathrm{C}=\mathrm{O}), 1632,1468,1223,856 \mathrm{~cm}^{-1} ;{ }^{1} \mathrm{H}$ NMR $\left(\mathrm{CDCl}_{3}\right) \delta 4.32-4.23\left(4 \mathrm{H}, \mathrm{m}, 2 \times \mathrm{OCH}_{2} \mathrm{CH}_{3}\right), 3.80\left(1 \mathrm{H}, \mathrm{m}, \mathrm{C}_{6}-\mathrm{H}\right)$, $3.08(1 \mathrm{H}, \mathrm{d}, J=15.6 \mathrm{~Hz}), 2.63(1 \mathrm{H}, \mathrm{d}, J=15.2 \mathrm{~Hz}), 2.41(1 \mathrm{H}, \mathrm{br} \mathrm{d}, J=16$ 
$\mathrm{Hz}), 2.31(1 \mathrm{H}, \mathrm{dd}, J=15.2,11.2 \mathrm{~Hz}), 1.79(1 \mathrm{H}, \mathrm{dd}, J=18,9.2 \mathrm{~Hz}), 1.60-1.38(3 \mathrm{H}, \mathrm{m}), 1.28$ $\left(6 \mathrm{H}, \mathrm{t}, J=7.2 \mathrm{~Hz}, 2 \times \mathrm{OCH}_{2} \mathrm{CH}_{3}\right), 0.94\left(3 \mathrm{H}, \mathrm{t}, J=7.2 \mathrm{~Hz}, \mathrm{CH}_{3}\right) ;{ }^{13} \mathrm{C} \mathrm{NMR}\left(\mathrm{CDCl}_{3}, \mathrm{DEPT}\right) \delta$ $202.5(\mathrm{C}, \mathrm{C}=\mathrm{O}), 167.5(\mathrm{C}, \mathrm{O}-\mathrm{C}=\mathrm{O}), 167.0(\mathrm{C}, \mathrm{O}-\mathrm{C}=\mathrm{O}), 81.4(\mathrm{C}, \mathrm{C}-2), 73.5(\mathrm{CH}, \mathrm{C}-6), 62.15$ $\left(\mathrm{CH}_{2}, \mathrm{OCH}_{2} \mathrm{CH}_{3}\right), 62.13\left(\mathrm{CH}_{2}, \mathrm{OCH}_{2} \mathrm{CH}_{3}\right), 46.1\left(\mathrm{CH}_{2}\right), 44.0\left(\mathrm{CH}_{2}\right), 37.7\left(\mathrm{CH}_{2}\right), 18.0\left(\mathrm{CH}_{2}\right)$, $13.73\left(\mathrm{CH}_{3}\right), 13.70\left(\mathrm{CH}_{3}, \mathrm{OCH}_{2} \mathrm{CH}_{3}\right), 13.4\left(\mathrm{CH}_{3}, \mathrm{OCH}_{2} \mathrm{CH}_{3}\right)$; HRMS (ESI-TOF): $\mathrm{m} / z$ $309.1319\left(\mathrm{M}+\mathrm{Na}^{+}\right)$, calcd for $\mathrm{C}_{14} \mathrm{H}_{22} \mathrm{O}_{6} \mathrm{Na}^{+}$309.1314; LCMS: $m / z 287.35(\mathrm{M}+1)$, calcd for $\mathrm{C}_{14} \mathrm{H}_{22} \mathrm{O}_{6} 286.1416$.

6-Butyl-4-oxotetrahydropyran-2,2-dicarboxylic acid diethyl ester (6h). Purified by

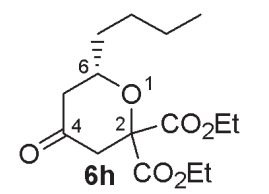
column chromatography using EtOAc/hexane and isolated as oil. IR (neat): $v_{\max } 2959,1745(\mathrm{C}=\mathrm{O}, \mathrm{O}-\mathrm{C}=\mathrm{O}), 1628,1468,1369,1215,1120,1068,854$, $783,667 \mathrm{~cm}^{-1} ;{ }^{1} \mathrm{H}$ NMR $\left(\mathrm{CDCl}_{3}\right) \delta 4.26\left(4 \mathrm{H}, \mathrm{m}, 2 \times \mathrm{OCH}_{2} \mathrm{CH}_{3}\right), 3.79(1 \mathrm{H}$, m, $\left.\mathrm{C}_{6}-\mathrm{H}\right), 3.08(1 \mathrm{H}, \mathrm{d}, J=15.6 \mathrm{~Hz}), 2.64(1 \mathrm{H}, \mathrm{d}, J=15.2 \mathrm{~Hz}), 2.42(1 \mathrm{H}, \mathrm{br}$ d, $J=14 \mathrm{~Hz}), 2.31(1 \mathrm{H}, \mathrm{dd}, J=15.2,11.6 \mathrm{~Hz}), 1.80(1 \mathrm{H}, \mathrm{m}), 1.60-1.40(2 \mathrm{H}, \mathrm{m}), 1.39-1.31$ $(3 \mathrm{H}, \mathrm{m}), 1.28\left(6 \mathrm{H}\right.$, br t, $\left.J=6.8 \mathrm{~Hz}, 2 \times \mathrm{OCH}_{2} \mathrm{CH}_{3}\right), 0.91\left(3 \mathrm{H}, \mathrm{t}, J=6.8 \mathrm{~Hz}, \mathrm{CH}_{3}\right) ;{ }^{13} \mathrm{C} \mathrm{NMR}$ $\left(\mathrm{CDCl}_{3}, \mathrm{DEPT}\right) \delta 202.9(\mathrm{C}, \mathrm{C}=\mathrm{O}), 167.7(\mathrm{C}, \mathrm{O}-\mathrm{C}=\mathrm{O}), 167.2(\mathrm{C}, \mathrm{O}-\mathrm{C}=\mathrm{O}), 82.1(\mathrm{C}, \mathrm{C}-2), 74.1$ (CH, C-6), $62.40\left(\mathrm{CH}_{2}, \mathrm{OCH}_{2} \mathrm{CH}_{3}\right), 62.39\left(\mathrm{CH}_{2}, \mathrm{OCH}_{2} \mathrm{CH}_{3}\right), 46.3\left(\mathrm{CH}_{2}\right), 44.3\left(\mathrm{CH}_{2}\right), 35.6$ $\left(\mathrm{CH}_{2}\right), 27.1\left(\mathrm{CH}_{2}\right), 22.3\left(\mathrm{CH}_{2}\right), 13.97\left(\mathrm{CH}_{3}\right), 13.92\left(\mathrm{CH}_{3}, \mathrm{OCH}_{2} \mathrm{CH}_{3}\right), 13.90\left(\mathrm{CH}_{3}\right.$, $\mathrm{OCH}_{2} \mathrm{CH}_{3}$ ); HRMS (ESI-TOF): $m / z$ 323.1481 $\left(\mathrm{M}+\mathrm{Na}^{+}\right.$), calcd for $\mathrm{C}_{15} \mathrm{H}_{24} \mathrm{O}_{6} \mathrm{Na}^{+} 323.1471$; LCMS: $m / z 301.40(\mathrm{M}+1)$, calcd for $\mathrm{C}_{15} \mathrm{H}_{24} \mathrm{O}_{6} 300.1573$.

4-Oxo-6-pentyltetrahydropyran-2,2-dicarboxylic acid diethyl ester (6i). Purified by

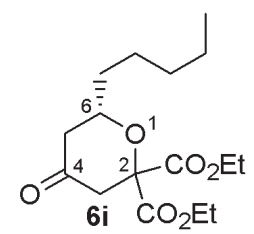
column chromatography using EtOAc/hexane and isolated as oil. IR (neat): $v_{\max } 2957,1744(\mathrm{C}=\mathrm{O}, \mathrm{O}-\mathrm{C}=\mathrm{O}), 1624,1466,1369,1279,1213,1067,858$ $\mathrm{cm}^{-1} ;{ }^{1} \mathrm{H} \mathrm{NMR}\left(\mathrm{CDCl}_{3}\right) \delta 4.29-4.26\left(4 \mathrm{H}\right.$, br q, $\left.J=6.8 \mathrm{~Hz}, 2 \times \mathrm{OCH}_{2} \mathrm{CH}_{3}\right)$, $3.80-3.78\left(1 \mathrm{H}, \mathrm{m}, \mathrm{C}_{6}-H\right), 3.08(1 \mathrm{H}, \mathrm{d}, J=15.6 \mathrm{~Hz}), 2.63(1 \mathrm{H}, \mathrm{d}, J=15.6$ $\mathrm{Hz}), 2.42(1 \mathrm{H}$, br d, $J=14 \mathrm{~Hz}), 2.30(1 \mathrm{H}, \mathrm{dd}, J=15.2,11.2 \mathrm{~Hz}), 1.78(1 \mathrm{H}$, m), 1.60-1.49 (2H, m), 1.30-1.27 (11H, m), $0.89\left(3 \mathrm{H}, \mathrm{t}, J=6.0 \mathrm{~Hz}, \mathrm{CH}_{3}\right) ;{ }^{13} \mathrm{C} \mathrm{NMR}\left(\mathrm{CDCl}_{3}\right.$, DEPT) $\delta 202.9(\mathrm{C}, \mathrm{C}=\mathrm{O}), 167.7(\mathrm{C}, \mathrm{O}-\mathrm{C}=\mathrm{O}), 167.2(\mathrm{C}, \mathrm{O}-\mathrm{C}=\mathrm{O}), 82.0(\mathrm{C}, \mathrm{C}-2), 74.0(\mathrm{CH}, \mathrm{C}-$ 6), $62.4\left(\mathrm{CH}_{2}, \mathrm{OCH}_{2} \mathrm{CH}_{3}\right), 62.3\left(\mathrm{CH}_{2}, \mathrm{OCH}_{2} \mathrm{CH}_{3}\right), 46.3\left(\mathrm{CH}_{2}\right), 44.3\left(\mathrm{CH}_{2}\right), 35.8\left(\mathrm{CH}_{2}\right), 31.4$ $\left(\mathrm{CH}_{2}\right), 24.6\left(\mathrm{CH}_{2}\right), 22.4\left(\mathrm{CH}_{2}\right), 13.94\left(\mathrm{CH}_{3}\right), 13.93\left(\mathrm{CH}_{3}, \mathrm{OCH}_{2} \mathrm{CH}_{3}\right), 13.89\left(\mathrm{CH}_{3}\right.$, $\mathrm{OCH}_{2} \mathrm{CH}_{3}$ ); HRMS (ESI-TOF): $m / z 337.1625\left(\mathrm{M}+\mathrm{Na}^{+}\right.$), calcd for $\mathrm{C}_{16} \mathrm{H}_{26} \mathrm{O}_{6} \mathrm{Na}^{+} 337.1627$.

6,6-Dimethyl-4-oxo-tetrahydropyran-2,2-dicarboxylic acid diethyl ester (6j). Purified by

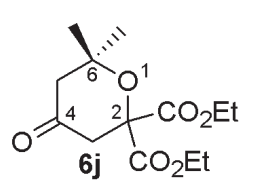

column chromatography using EtOAc/hexane and isolated as oil. IR (neat): $v_{\max } 2982,1742(\mathrm{C}=\mathrm{O}, \mathrm{O}-\mathrm{C}=\mathrm{O}), 1622,1467,1369,1215,1066,945,860$, $791 \mathrm{~cm}^{-1} ;{ }^{1} \mathrm{H}$ NMR $\left(\mathrm{CDCl}_{3}\right) \delta 4.26-4.20(4 \mathrm{H}$, br q, $J=7.2 \mathrm{~Hz}, 2 \times$ $\left.\mathrm{OCH}_{2} \mathrm{CH}_{3}\right), 2.94(2 \mathrm{H}, \mathrm{s}), 2.46(2 \mathrm{H}, \mathrm{s}), 1.34\left(6 \mathrm{H}, \mathrm{br} \mathrm{s}, 2 \times \mathrm{CH}_{3}\right), 1.25(6 \mathrm{H}$, br t, $\left.J=6.8 \mathrm{~Hz}, 2 \times \mathrm{OCH}_{2} \mathrm{CH}_{3}\right) ;{ }^{13} \mathrm{C} \mathrm{NMR}\left(\mathrm{CDCl}_{3}\right.$, DEPT) $\delta 203.4(\mathrm{C}, \mathrm{C}=\mathrm{O}), 168.9(2 \times \mathrm{C}$, $\mathrm{O}-\mathrm{C}=\mathrm{O}), 80.2(\mathrm{C}, \mathrm{C}-2), 77.3(\mathrm{C}, \mathrm{C}-6), 62.4\left(2 \times \mathrm{CH}_{2}, \mathrm{OCH}_{2} \mathrm{CH}_{3}\right), 50.9\left(\mathrm{CH}_{2}\right), 42.3\left(\mathrm{CH}_{2}\right)$, $29.3\left(2 \times \mathrm{CH}_{3}, \mathrm{C}_{6}-\mathrm{CH}_{3}\right), 13.8\left(2 \times \mathrm{CH}_{3}, 2 \times \mathrm{OCH}_{2} \mathrm{CH}_{3}\right)$; HRMS (ESI-TOF): $m / z 295.1153(\mathrm{M}$ $+\mathrm{Na}^{+}$), calcd for $\mathrm{C}_{13} \mathrm{H}_{20} \mathrm{O}_{6} \mathrm{Na}^{+} 295.1158$. 
6-(4-Methoxyphenyl)-4-oxotetrahydropyran-2,2-dicarboxylic acid diethyl ester (6k).

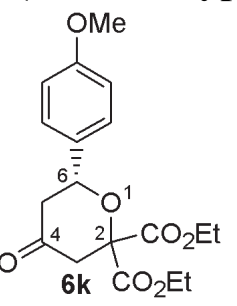

Purified by column chromatography using EtOAc/hexane and isolated as oil. IR (neat): $v_{\max }$ 2986, $1747(\mathrm{C}=\mathrm{O}, \mathrm{O}-\mathrm{C}=\mathrm{O}), 1601,1514,1468,1371,1246$, 1028, 858, 833, $723 \mathrm{~cm}^{-1} ;{ }^{1} \mathrm{H}$ NMR $\left(\mathrm{CDCl}_{3}\right) \delta 7.34(2 \mathrm{H}, \mathrm{d}, J=8.4 \mathrm{~Hz}), 6.92$ $(2 \mathrm{H}, \mathrm{d}, J=8.4 \mathrm{~Hz})[\mathrm{Ph}-H] ; 4.90\left(1 \mathrm{H}, \mathrm{dd}, J=10.4,4.4 \mathrm{~Hz}, \mathrm{C}_{6}-\mathrm{H}\right), 4.35-4.27$ $\left(4 \mathrm{H}, \mathrm{m}, 2 \times \mathrm{OCH}_{2} \mathrm{CH}_{3}\right), 3.81\left(3 \mathrm{H}, \mathrm{s}, \mathrm{OCH}_{3}\right), 3.17(1 \mathrm{H}, \mathrm{d}, J=16.0 \mathrm{~Hz}), 2.84$ $(1 \mathrm{H}, \mathrm{d}, J=16.0 \mathrm{~Hz}), 2.68(2 \mathrm{H}, \mathrm{m}), 1.32\left(6 \mathrm{H}, \mathrm{t}, J=7.2 \mathrm{~Hz}, 2 \times \mathrm{OCH}_{2} \mathrm{CH}_{3}\right)$; ${ }^{13} \mathrm{C}$ NMR $\left(\mathrm{CDCl}_{3}\right.$, DEPT) $\delta 202.6(\mathrm{C}, \mathrm{C}=\mathrm{O}), 167.7(\mathrm{C}, \mathrm{O}=\mathrm{C}-\mathrm{O}), 167.1(\mathrm{C}, \mathrm{O}=\mathrm{C}-\mathrm{O}), 159.7$ (C, $C$-OMe), $130.0(\mathrm{C}), 127.4(2 \times \mathrm{CH}, \mathrm{Ph}-\mathrm{CH}), 114.4(2 \times \mathrm{CH}, \mathrm{Ph}-\mathrm{CH}), 82.1(\mathrm{C}, \mathrm{C}-2), 75.3$ $(\mathrm{CH}, \mathrm{C}-6), 63.4\left(\mathrm{CH}_{2}, \mathrm{OCH}_{2} \mathrm{CH}_{3}\right), 63.3\left(\mathrm{CH}_{2}, \mathrm{OCH}_{2} \mathrm{CH}_{3}\right), 55.4\left(\mathrm{CH}_{3}, \mathrm{OCH}_{3}\right), 47.7\left(\mathrm{CH}_{2}\right)$, $44.8\left(\mathrm{CH}_{2}\right), 14.0\left(\mathrm{CH}_{3}, \mathrm{OCH}_{2} \mathrm{CH}_{3}\right), 13.8\left(\mathrm{CH}_{3}, \mathrm{OCH}_{2} \mathrm{CH}_{3}\right)$; LRMS: $m / z 351.15(\mathrm{M}+1)$, calcd for $\mathrm{C}_{18} \mathrm{H}_{22} \mathrm{O}_{7} 351.3631$.

2-Hydroxy-2-[4-(4-methoxyphenyl)-2-oxobut-3-enyl]malonic acid diethyl ester (7k).

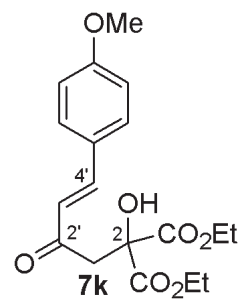

Purified by column chromatography using EtOAc/hexane and isolated as oil.

IR (neat): $v_{\max } 3464(\mathrm{O}-\mathrm{H}), 2986,1747(\mathrm{C}=\mathrm{O}, \mathrm{O}-\mathrm{C}=\mathrm{O}), 1601,1514,1468$, $1371,1246,1028,858,833,723 \mathrm{~cm}^{-1} ;{ }^{1} \mathrm{H} \mathrm{NMR}\left(\mathrm{CDCl}_{3}\right) \delta 7.56(1 \mathrm{H}, \mathrm{d}, J=$

$16.0 \mathrm{~Hz}$, olefinic- $\beta-\mathrm{H}), 7.50(2 \mathrm{H}, \mathrm{d}, J=8.8 \mathrm{~Hz}), 6.92(2 \mathrm{H}, \mathrm{d}, J=8.8 \mathrm{~Hz})$

$[\mathrm{Ph}-H] ; 6.61(1 \mathrm{H}, \mathrm{d}, J=15.6 \mathrm{~Hz}$, olefinic- $\alpha-\mathrm{H}), 4.38-4.26(4 \mathrm{H}, \mathrm{m}, 2 \times$ $\left.\mathrm{OCH}_{2} \mathrm{CH}_{3}\right), 3.85\left(3 \mathrm{H}, \mathrm{s}, \mathrm{OCH}_{3}\right), 3.51\left(2 \mathrm{H}, \mathrm{s}, \mathrm{CH}_{2}\right), 1.30(6 \mathrm{H}, \mathrm{t}, J=7.2 \mathrm{~Hz}, 2$ $\left.\times \mathrm{OCH}_{2} \mathrm{CH}_{3}\right) ;{ }^{13} \mathrm{C} \mathrm{NMR}\left(\mathrm{CDCl}_{3}, \mathrm{DEPT}\right) \delta 196.3(\mathrm{C}, \mathrm{C}=\mathrm{O}), 169.6(2 \times \mathrm{C}, \mathrm{O}=\mathrm{C}-\mathrm{O}), 161.9(\mathrm{C}$, $\left.C-\mathrm{OCH}_{3}\right), 144.0(\mathrm{CH}$, olefinic- $\beta-\mathrm{CH}), 130.2(2 \times \mathrm{CH}, \mathrm{Ph}-\mathrm{CH}), 126.7(\mathrm{C}), 123.5(\mathrm{CH}$, olefinic- $\alpha-C H), 114.4(2 \times \mathrm{CH}, \mathrm{Ph}-\mathrm{CH}), 62.6\left(2 \times \mathrm{CH}_{2}, \mathrm{OCH}_{2} \mathrm{CH}_{3}\right), 55.4\left(\mathrm{CH}_{3}, \mathrm{OCH}_{3}\right), 44.8$ $\left(\mathrm{CH}_{2}\right), 13.9\left(2 \times \mathrm{CH}_{3}, \mathrm{OCH}_{2} \mathrm{CH}_{3}\right)$; LRMS: $m / z 351.15(\mathrm{M}+1)$, calcd for $\mathrm{C}_{18} \mathrm{H}_{22} \mathrm{O}_{7} 351.3631$.

6-(4-Fluorophenyl)-4-oxotetrahydropyran-2,2-dicarboxylic acid diethyl ester (61).

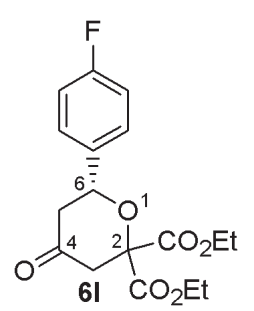

Purified by column chromatography using EtOAc/hexane and isolated as oil. IR (neat): $v_{\max } 2926,1747(\mathrm{C}=\mathrm{O}, \mathrm{O}-\mathrm{C}=\mathrm{O}), 1603(\mathrm{C}=\mathrm{C}), 1508,1364,1190$, $1082,849,779,663 \mathrm{~cm}^{-1} ;{ }^{1} \mathrm{H} \mathrm{NMR}\left(\mathrm{CDCl}_{3}\right) \delta 7.42-7.39(2 \mathrm{H}, \mathrm{dd}, J=8.4,5.2$ $\mathrm{Hz}), 7.07(2 \mathrm{H}, \mathrm{t}, J=8.8 \mathrm{~Hz})[\mathrm{Ph}-H] ; 4.95\left(1 \mathrm{H}, \mathrm{dd}, J=9.6,4.8 \mathrm{~Hz}, \mathrm{C}_{6}-\mathrm{H}\right)$, 4.31-4.28 $\left(4 \mathrm{H}, \mathrm{m}, 2 \times \mathrm{OCH}_{2} \mathrm{CH}_{3}\right), 3.18(1 \mathrm{H}, \mathrm{d}, J=15.2 \mathrm{~Hz}), 2.85(1 \mathrm{H}, \mathrm{d}, J=$ $15.2 \mathrm{~Hz}), 2.66-2.63(2 \mathrm{H}, \mathrm{m}), 1.29\left(6 \mathrm{H}, \mathrm{t}, J=7.2 \mathrm{~Hz}, 2 \times \mathrm{OCH}_{2} \mathrm{CH}_{3}\right) ;{ }^{13} \mathrm{C}$ NMR ( $\mathrm{CDCl}_{3}$, DEPT) $\delta 201.9(\mathrm{C}, \mathrm{C}=\mathrm{O}), 169.5(\mathrm{C}, \mathrm{O}=\mathrm{C}-\mathrm{O}), 166.9(\mathrm{C}, \mathrm{O}=\mathrm{C}-\mathrm{O}), 162.6(\mathrm{C}, \mathrm{d}$, $J=245.6 \mathrm{~Hz}, C-\mathrm{F}), 135.2(\mathrm{C}, \mathrm{d}, J=3.0 \mathrm{~Hz}, \mathrm{C}-7), 127.7(2 \times \mathrm{CH}, \mathrm{d}, J=8.2 \mathrm{~Hz}), 115.5(2 \times$ $\mathrm{CH}, \mathrm{d}, J=21.5 \mathrm{~Hz}), 82.1(\mathrm{C}, \mathrm{C}-2), 74.9(\mathrm{CH}, \mathrm{C}-6), 62.65\left(\mathrm{CH}_{2}, \mathrm{OCH}_{2} \mathrm{CH}_{3}\right), 62.60\left(\mathrm{CH}_{2}\right.$, $\left.\mathrm{OCH}_{2} \mathrm{CH}_{3}\right), 47.8\left(\mathrm{CH}_{2}\right), 44.1\left(\mathrm{CH}_{2}\right), 13.9\left(\mathrm{CH}_{3}, \mathrm{OCH}_{2} \mathrm{CH}_{3}\right), 13.8\left(\mathrm{CH}_{3}, \mathrm{OCH}_{2} \mathrm{CH}_{3}\right)$; LRMS: $m / z 339.20(\mathrm{M}+1)$, calcd for $\mathrm{C}_{17} \mathrm{H}_{19} \mathrm{FO}_{6} 338.1166$.

2-[4-(4-Fluorophenyl)-2-oxobut-3-enyl]-2-hydroxymalonic acid diethyl ester (7l).

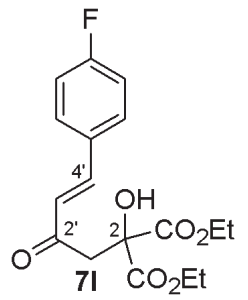

Purified by column chromatography using EtOAc/hexane and isolated as oil. IR (neat): $v_{\max } 3482(\mathrm{O}-\mathrm{H}), 2926,1747(\mathrm{C}=\mathrm{O}, \mathrm{O}-\mathrm{C}=\mathrm{O}), 1603(\mathrm{C}=\mathrm{C}), 1508$, 1364, 1190, 1082, 849, 779, $663 \mathrm{~cm}^{-1} ;{ }^{1} \mathrm{H} \mathrm{NMR}\left(\mathrm{CDCl}_{3}\right) \delta 7.56(1 \mathrm{H}, \mathrm{d}, J=$ $16.4 \mathrm{~Hz}), 7.53(2 \mathrm{H}, \mathrm{m}), 7.09(2 \mathrm{H}, \mathrm{t}, J=8.8 \mathrm{~Hz}), 6.66(1 \mathrm{H}, \mathrm{d}, J=16.4 \mathrm{~Hz})$, $4.29\left(4 \mathrm{H}, \mathrm{m}, 2 \times \mathrm{OCH}_{2} \mathrm{CH}_{3}\right), 3.52\left(2 \mathrm{H}, \mathrm{s}, \mathrm{CH}_{2}\right), 1.30(6 \mathrm{H}, \mathrm{t}, J=7.2 \mathrm{~Hz}, 2 \times$ $\left.\mathrm{OCH}_{2} \mathrm{CH}_{3}\right) ;{ }^{13} \mathrm{C} \mathrm{NMR}\left(\mathrm{CDCl}_{3}, \mathrm{DEPT}\right) \delta 195.9(\mathrm{C}, \mathrm{C}=\mathrm{O}), 167.5(2 \times \mathrm{C}$, $\mathrm{O}=\mathrm{C}-\mathrm{O}), 163.0(\mathrm{C}, \mathrm{d}, J=245.0 \mathrm{~Hz}, C-\mathrm{F}), 142.6(\mathrm{CH}), 136.0(\mathrm{C}), 130.3(2 \times \mathrm{CH}, \mathrm{d}, J=8.7$ 
$\mathrm{Hz}, \mathrm{Ph}-\mathrm{CH}), 125.4(\mathrm{CH}), 116.1(2 \times \mathrm{CH}, \mathrm{d}, J=21.8 \mathrm{~Hz}, \mathrm{Ph}-\mathrm{CH}), 76.8(\mathrm{C}, \mathrm{C}-\mathrm{OH}), 45.0$ $\left(\mathrm{CH}_{2}\right), 13.8\left(2 \times \mathrm{CH}_{3}, \mathrm{OCH}_{2} \mathrm{CH}_{3}\right)$; LRMS: $m / z 339.20(\mathrm{M}+1)$, calcd for $\mathrm{C}_{17} \mathrm{H}_{19} \mathrm{FO}_{6}$ 338.1166 .

6-(4-Chlorophenyl)-4-oxotetrahydropyran-2,2-dicarboxylic acid diethyl ester (6m).

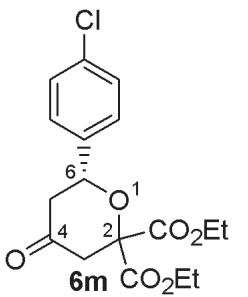

Purified by column chromatography using EtOAc/hexane and isolated as oil. IR (neat): $v_{\max } 2986,1742(\mathrm{C}=\mathrm{O}, \mathrm{O}-\mathrm{C}=\mathrm{O}), 1493,1369,1231,1088,1014$, 812, 737, $652 \mathrm{~cm}^{-1} ;{ }^{1} \mathrm{H}$ NMR $\left(\mathrm{CDCl}_{3}\right) \delta 7.48(2 \mathrm{H}, \mathrm{d}, J=8.0 \mathrm{~Hz}), 7.37(2 \mathrm{H}, \mathrm{d}$, $J=8.0 \mathrm{~Hz})[\mathrm{Ph}-H] ; 4.95\left(1 \mathrm{H}, \mathrm{dd}, J=10.8,3.2 \mathrm{~Hz}, \mathrm{C}_{6}-\mathrm{H}\right), 4.28(4 \mathrm{H}, \mathrm{m}, 2 \times$ $\left.\mathrm{OCH}_{2} \mathrm{CH}_{3}\right), 3.18(1 \mathrm{H}, \mathrm{d}, J=15.6 \mathrm{~Hz}), 2.85(1 \mathrm{H}, \mathrm{d}, J=15.6 \mathrm{~Hz}), 2.69-2.56$ $(2 \mathrm{H}, \mathrm{m}), 1.29\left(6 \mathrm{H}, \mathrm{t}, J=7.2 \mathrm{~Hz}, 2 \times \mathrm{OCH}_{2} \mathrm{CH}_{3}\right) ;{ }^{13} \mathrm{C} \mathrm{NMR}\left(\mathrm{CDCl}_{3}\right.$, DEPT $) \delta$ $201.7(\mathrm{C}, \mathrm{C}=\mathrm{O}), 167.4(\mathrm{C}, \mathrm{O}=\mathrm{C}-\mathrm{O}), 166.8(\mathrm{C}, \mathrm{O}=\mathrm{C}-\mathrm{O}), 137.8(\mathrm{C}), 132.5(\mathrm{C}), 129.2(2 \times \mathrm{CH}$, $\mathrm{Ph}-\mathrm{CH}), 127.2(2 \times \mathrm{CH}, \mathrm{Ph}-\mathrm{CH}), 82.1(\mathrm{C}, \mathrm{C}-2), 74.8(\mathrm{CH}, \mathrm{C}-6), 62.65\left(\mathrm{CH}_{2}, \mathrm{OCH}_{2} \mathrm{CH}_{3}\right)$, $62.62\left(\mathrm{CH}_{2}, \mathrm{OCH}_{2} \mathrm{CH}_{3}\right), 45.1\left(\mathrm{CH}_{2}\right), 44.1\left(\mathrm{CH}_{2}\right), 13.9\left(\mathrm{CH}_{3}, \mathrm{OCH}_{2} \mathrm{CH}_{3}\right), 13.8\left(\mathrm{CH}_{3}\right.$, $\left.\mathrm{OCH}_{2} \mathrm{CH}_{3}\right)$; LRMS: $\mathrm{m} / z$ 355.15 (M+1), calcd for $\mathrm{C}_{17} \mathrm{H}_{19} \mathrm{ClO}_{6} 354.0870$.

2-[4-(4-Chlorophenyl)-2-oxobut-3-enyl]-2-hydroxymalonic acid diethyl ester (7m).

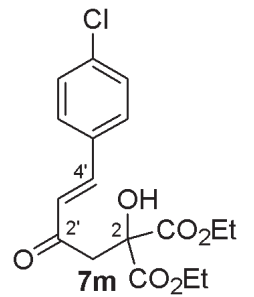

Purified by column chromatography using EtOAc/hexane and isolated as oil. IR (neat): $v_{\max } 3497(\mathrm{O}-\mathrm{H}), 2986,1742(\mathrm{C}=\mathrm{O}, \mathrm{O}-\mathrm{C}=\mathrm{O}), 1665(\mathrm{C}=\mathrm{C}-\mathrm{C}=\mathrm{O})$, $1613(\mathrm{C}=\mathrm{C}), 1493,1369,1231,1088,1014,812,737,652 \mathrm{~cm}^{-1} ;{ }^{1} \mathrm{H}$ NMR $\left(\mathrm{CDCl}_{3}\right) \delta 7.52(1 \mathrm{H}, \mathrm{d}, J=15.6 \mathrm{~Hz}$, olefinic-H), $7.36(4 \mathrm{H}$, br s, Ph- $H), 6.70$ $(1 \mathrm{H}, \mathrm{d}, J=15.6 \mathrm{~Hz}$, olefinic- $H), 4.29\left(4 \mathrm{H}, \mathrm{m}, 2 \times \mathrm{OCH}_{2} \mathrm{CH}_{3}\right), 3.52(2 \mathrm{H}, \mathrm{s}$, $\left.\mathrm{CH}_{2}\right), 1.30\left(6 \mathrm{H}, \mathrm{t}, J=7.2 \mathrm{~Hz}, 2 \times \mathrm{OCH}_{2} \mathrm{CH}_{3}\right) ;{ }^{13} \mathrm{C} \mathrm{NMR}\left(\mathrm{CDCl}_{3}, \mathrm{DEPT}\right) \delta$ $195.9(\mathrm{C}, \mathrm{C}=\mathrm{O}), 169.4(2 \times \mathrm{C}, \mathrm{O}=\mathrm{C}-\mathrm{O}), 142.4(\mathrm{CH}$, olefinic-CH$), 136.7(\mathrm{C}), 134.2(\mathrm{C}), 129.5$ $(2 \times \mathrm{CH}, \mathrm{Ph}-\mathrm{CH}), 128.8(2 \times \mathrm{CH}, \mathrm{Ph}-\mathrm{CH}), 126.1(\mathrm{CH}$, olefinic- $\mathrm{CH}), 76.7(\mathrm{C}, \mathrm{C}-\mathrm{OH}), 62.6(2$ $\left.\times \mathrm{CH}_{2}, \mathrm{OCH}_{2} \mathrm{CH}_{3}\right), 47.6\left(\mathrm{CH}_{2}\right), 13.8\left(2 \times \mathrm{CH}_{3}, \mathrm{OCH}_{2} \mathrm{CH}_{3}\right)$; LRMS: $m / z 355.15(\mathrm{M}+1)$, calcd for $\mathrm{C}_{17} \mathrm{H}_{19} \mathrm{ClO}_{6} 354.0870$.

6-(4-Bromophenyl)-4-oxotetrahydropyran-2,2-dicarboxylic acid diethyl ester (6n).

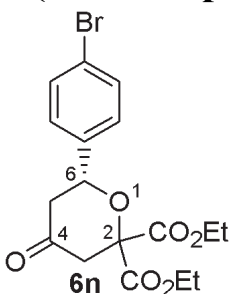

Purified by column chromatography using EtOAc/hexane and isolated as oil. IR (neat): $v_{\max } 2980,1740(\mathrm{C}=\mathrm{O}, \mathrm{O}-\mathrm{C}=\mathrm{O}), 1653,1582,1489,1286,1232$, $1072,1009,810,640 \mathrm{~cm}^{-1} ;{ }^{1} \mathrm{H}$ NMR $\left(\mathrm{CDCl}_{3}\right) \delta 7.53(2 \mathrm{H}, \mathrm{d}, J=8.4 \mathrm{~Hz}), 7.30$ $(2 \mathrm{H}, \mathrm{d}, J=8.4 \mathrm{~Hz})[\mathrm{Ph}-H] ; 4.94\left(1 \mathrm{H}, \mathrm{dd}, J=10.8,5.2 \mathrm{~Hz}, \mathrm{C}_{6}-\mathrm{H}\right), 4.32-4.27$ $\left(4 \mathrm{H}, \mathrm{m}, 2 \times \mathrm{OCH}_{2} \mathrm{CH}_{3}\right), 3.17(1 \mathrm{H}, \mathrm{d}, J=16.0 \mathrm{~Hz}), 2.85(1 \mathrm{H}, \mathrm{d}, J=15.6 \mathrm{~Hz})$, 2.69-2.57 (2H, m), 1.32-1.26 (6H, m, $\left.2 \times \mathrm{OCH}_{2} \mathrm{CH}_{3}\right) ;{ }^{13} \mathrm{C} \mathrm{NMR}\left(\mathrm{CDCl}_{3}\right.$, DEPT) $\delta 201.7(\mathrm{C}, \mathrm{C}=\mathrm{O}), 167.4(\mathrm{C}, \mathrm{O}=\mathrm{C}-\mathrm{O}), 166.8(\mathrm{C}, \mathrm{O}=\mathrm{C}-\mathrm{O}), 138.4(\mathrm{C}, \mathrm{C}-\mathrm{Br}), 131.8$ (2 $\times$ $\mathrm{CH}, \mathrm{Ph}-\mathrm{CH}), 127.6(2 \times \mathrm{CH}, \mathrm{Ph}-\mathrm{CH}), 122.3(\mathrm{C}), 82.1$ (C, C-2), $74.8(\mathrm{CH}, \mathrm{C}-6), 62.7(2 \times$ $\left.\mathrm{CH}_{2}, \mathrm{OCH}_{2} \mathrm{CH}_{3}\right), 47.6\left(\mathrm{CH}_{2}\right), 44.1\left(\mathrm{CH}_{2}\right), 13.94\left(\mathrm{CH}_{3}, \mathrm{OCH}_{2} \mathrm{CH}_{3}\right), 13.90\left(\mathrm{CH}_{3}, \mathrm{OCH}_{2} \mathrm{CH}_{3}\right)$; LRMS: $m / z 421.05\left(\mathrm{M}+\mathrm{Na}^{+}\right)$, calcd for $\mathrm{C}_{17} \mathrm{H}_{19} \mathrm{BrO}_{6} \mathrm{Na}^{+} 421.0262$.

2-[4-(4-Bromophenyl)-2-oxobut-3-enyl]-2-hydroxymalonic acid diethyl ester (7n).

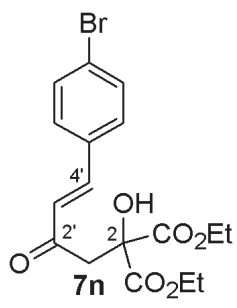
Purified by column chromatography using EtOAc/hexane and isolated as oil. IR (neat): $v_{\max } 3420(\mathrm{O}-\mathrm{H}), 2980,1740(\mathrm{C}=\mathrm{O}, \mathrm{O}-\mathrm{C}=\mathrm{O}), 1653,1582$, $1489,1286,1232,1072,1009,810,640 \mathrm{~cm}^{-1} ;{ }^{1} \mathrm{H}$ NMR $\left(\mathrm{CDCl}_{3}\right) \delta 7.54(1 \mathrm{H}$, $\mathrm{d}, J=16.0 \mathrm{~Hz}$, olefinic- $\beta-\mathrm{H}), 7.53(2 \mathrm{H}, \mathrm{d}, J=8.4 \mathrm{~Hz}), 7.41(2 \mathrm{H}, \mathrm{t}, J=8.0$ $\mathrm{Hz}), 6.71(1 \mathrm{H}, \mathrm{d}, J=16.0 \mathrm{~Hz}$, olefinic- $\alpha-\mathrm{H}), 4.32-4.27(4 \mathrm{H}, \mathrm{m}, 2 \times$ $\left.\mathrm{OCH}_{2} \mathrm{CH}_{3}\right), 3.52\left(2 \mathrm{H}, \mathrm{s}, \mathrm{CH}_{2}\right), 1.32-1.26\left(6 \mathrm{H}, \mathrm{m}, 2 \times \mathrm{OCH}_{2} \mathrm{CH}_{3}\right) ;{ }^{13} \mathrm{C} \mathrm{NMR}$ 
$\left(\mathrm{CDCl}_{3}, \mathrm{DEPT}\right) \delta 195.9(\mathrm{C}, \mathrm{C}=\mathrm{O}), 169.4(2 \times \mathrm{C}, \mathrm{O}=\mathrm{C}-\mathrm{O}), 142.6(\mathrm{CH}$, olefinic-CH$), 132.9(\mathrm{C}$, $\mathrm{C}-\mathrm{Br}), 132.2(2 \times \mathrm{CH}, \mathrm{Ph}-\mathrm{CH}), 129.7(2 \times \mathrm{CH}, \mathrm{Ph}-\mathrm{CH}), 126.2(\mathrm{CH}$, olefinic-CH $), 125.1(\mathrm{C})$, $76.7(\mathrm{C}, \mathrm{C}-\mathrm{OH}), 62.6\left(2 \times \mathrm{CH}_{2}, \mathrm{OCH}_{2} \mathrm{CH}_{3}\right), 45.1\left(\mathrm{CH}_{2}\right), 13.89\left(2 \times \mathrm{CH}_{3}, \mathrm{OCH}_{2} \mathrm{CH}_{3}\right)$; LRMS: $m / z 421.05\left(\mathrm{M}+\mathrm{Na}^{+}\right)$, calcd for $\mathrm{C}_{17} \mathrm{H}_{19} \mathrm{BrO}_{6} \mathrm{Na}^{+} 421.0262$.

6-(4-Cyanophenyl)-4-oxotetrahydropyran-2,2-dicarboxylic acid diethyl ester (60). Purified by column chromatography using EtOAc/hexane and isolated as oil. IR (neat): $v_{\max }$

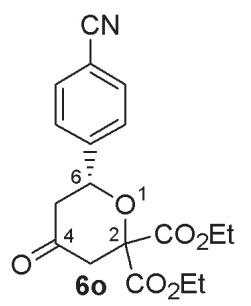
2984, $2229(\mathrm{C} \equiv \mathrm{N}), 1740(\mathrm{C}=\mathrm{O}, \mathrm{O}-\mathrm{C}=\mathrm{O}), 1612,1506,1468,1369,1283$, 1227, 1072, 1018, $856 \mathrm{~cm}^{-1} ;{ }^{1} \mathrm{H}$ NMR $\left(\mathrm{CDCl}_{3}\right) \delta 7.68(2 \mathrm{H}, \mathrm{d}, J=7.6 \mathrm{~Hz})$, $7.54(2 \mathrm{H}, \mathrm{d}, J=7.6 \mathrm{~Hz})[\mathrm{Ph}-H] ; 5.04\left(1 \mathrm{H}, \mathrm{br} \mathrm{d}, J=11.6 \mathrm{~Hz}, \mathrm{C}_{6}-\mathrm{H}\right), 4.30$ $\left(4 \mathrm{H}, \mathrm{m}, 2 \times \mathrm{OCH}_{2} \mathrm{CH}_{3}\right), 3.17(1 \mathrm{H}, \mathrm{d}, J=15.6 \mathrm{~Hz}), 2.86(1 \mathrm{H}, \mathrm{d}, J=15.6$ $\mathrm{Hz}), 2.70(1 \mathrm{H}, \mathrm{d}, J=14.4 \mathrm{~Hz}), 2.56(1 \mathrm{H}, \mathrm{dd}, J=14.4,11.6 \mathrm{~Hz}), 1.28(6 \mathrm{H}, \mathrm{t}$, $\left.J=7.2 \mathrm{~Hz}, 2 \times \mathrm{OCH}_{2} \mathrm{CH}_{3}\right) ;{ }^{13} \mathrm{C} \mathrm{NMR}\left(\mathrm{CDCl}_{3}\right.$, DEPT $) \delta 201.1(\mathrm{C}, \mathrm{C}=\mathrm{O})$, $167.2(\mathrm{C}, \mathrm{O}=\mathrm{C}-\mathrm{O}), 166.7(\mathrm{C}, \mathrm{O}=\mathrm{C}-\mathrm{O}), 144.5(\mathrm{C}, \mathrm{C}-\mathrm{CN}), 132.5(2 \times \mathrm{CH}, \mathrm{Ph}-\mathrm{CH}), 126.4(2 \times$ $\mathrm{CH}, \mathrm{Ph}-\mathrm{CH}), 118.4(\mathrm{C}), 112.2(\mathrm{C}, \mathrm{C} \equiv \mathrm{N}), 82.1(\mathrm{C}, \mathrm{C}-2), 74.5(\mathrm{CH}, \mathrm{C}-6), 62.8\left(2 \times \mathrm{CH}_{2}\right.$, $\left.\mathrm{OCH}_{2} \mathrm{CH}_{3}\right), 47.4\left(\mathrm{CH}_{2}\right), 44.1\left(\mathrm{CH}_{2}\right), 13.95\left(\mathrm{CH}_{3}, \mathrm{OCH}_{2} \mathrm{CH}_{3}\right), 13.92\left(\mathrm{CH}_{3}, \mathrm{OCH}_{2} \mathrm{CH}_{3}\right)$; HRMS (ESI-TOF): $m / z 368.1118\left(\mathrm{M}+\mathrm{Na}^{+}\right)$, calcd for $\mathrm{C}_{18} \mathrm{H}_{19} \mathrm{NO}_{6} \mathrm{Na}^{+} 368.1110$.

2-[4-(4-Cyanophenyl)-2-oxobut-3-enyl]-2-hydroxymalonic acid diethyl ester (70). Purified by column chromatography using EtOAc/hexane and isolated as oil. IR (neat): $v_{\max }$

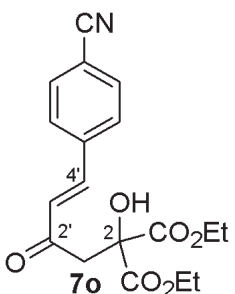
$3458(\mathrm{O}-\mathrm{H}), 2984,2229(\mathrm{C} \equiv \mathrm{N}), 1740(\mathrm{C}=\mathrm{O}, \mathrm{O}-\mathrm{C}=\mathrm{O}), 1612,1506,1468$, $1369,1283,1227,1072,1018,856 \mathrm{~cm}^{-1} ;{ }^{1} \mathrm{H}$ NMR $\left(\mathrm{CDCl}_{3}\right) \delta 7.69-7.53$ $(5 \mathrm{H}, \mathrm{m}, \mathrm{Ph}-H$, olefinic- $\beta-\mathrm{H}), 6.78(1 \mathrm{H}, \mathrm{d}, J=16.0 \mathrm{~Hz}$, olefinic- $\alpha-\mathrm{H}), 4.39-$ $4.22\left(4 \mathrm{H}, \mathrm{m}, 2 \times \mathrm{OCH}_{2} \mathrm{CH}_{3}\right), 3.52\left(2 \mathrm{H}, \mathrm{s}, \mathrm{CH}_{2}\right), 1.28(6 \mathrm{H}, \mathrm{t}, J=7.2 \mathrm{~Hz}, 2 \times$ $\left.\mathrm{OCH}_{2} \mathrm{CH}_{3}\right) ;{ }^{13} \mathrm{C} \mathrm{NMR}\left(\mathrm{CDCl}_{3}\right.$, DEPT) $\delta 195.5(\mathrm{C}, \mathrm{C}=\mathrm{O}), 169.4(2 \times \mathrm{C}$, $\mathrm{O}=\mathrm{C}-\mathrm{O}), 141.2(\mathrm{CH}$, olefinic- $\beta-\mathrm{CH}), 138.4(\mathrm{C}), 132.6(2 \times \mathrm{CH}, \mathrm{Ph}-\mathrm{CH})$, $128.7(2 \times \mathrm{CH}, \mathrm{Ph}-\mathrm{CH}), 128.5(\mathrm{CH}$, olefinic- $\alpha-\mathrm{CH}), 118.2(\mathrm{C}), 113.8(\mathrm{C}, \mathrm{CN}), 76.7(\mathrm{C}, C-$ $\mathrm{OH}), 62.8\left(2 \times \mathrm{CH}_{2}, \mathrm{OCH}_{2} \mathrm{CH}_{3}\right), 45.4\left(\mathrm{CH}_{2}\right), 13.9\left(2 \times \mathrm{CH}_{3}, \mathrm{OCH}_{2} \mathrm{CH}_{3}\right) ; \mathrm{HRMS}(\mathrm{ESI}-\mathrm{TOF})$ : $m / z 368.1118\left(\mathrm{M}+\mathrm{Na}^{+}\right)$, calcd for $\mathrm{C}_{18} \mathrm{H}_{19} \mathrm{NO}_{6} \mathrm{Na}^{+} 368.1110$.

6-(4-Nitrophenyl)-4-oxotetrahydropyran-2,2-dicarboxylic acid diethyl ester (6p). Purified by column chromatography using EtOAc/hexane and isolated as oil. IR (neat): $v_{\max }$

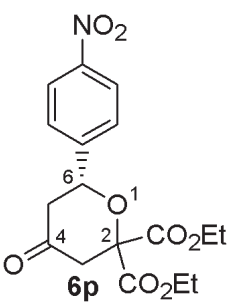
2938, $1742(\mathrm{C}=\mathrm{O}, \mathrm{O}-\mathrm{C}=\mathrm{O}), 1603,1524,1468,1348,1290,1227,1165$, $1072,1014,856,748,698,646 \mathrm{~cm}^{-1} ;{ }^{1} \mathrm{H} \mathrm{NMR}\left(\mathrm{CDCl}_{3}\right) \delta 8.26(2 \mathrm{H}, \mathrm{d}, J=$ $8.4 \mathrm{~Hz}), 7.62(2 \mathrm{H}, \mathrm{d}, J=8.4 \mathrm{~Hz})[\mathrm{Ph}-H] ; 5.13\left(1 \mathrm{H}, \mathrm{br} \mathrm{d}, J=11.6 \mathrm{~Hz}, \mathrm{C}_{6^{-}}\right.$ $\mathrm{H}), 4.34-4.26\left(4 \mathrm{H}, \mathrm{m}, 2 \times \mathrm{OCH}_{2} \mathrm{CH}_{3}\right), 3.20(1 \mathrm{H}, \mathrm{d}, J=15.6 \mathrm{~Hz}), 2.90(1 \mathrm{H}$, $\mathrm{d}, J=15.6 \mathrm{~Hz}), 2.75(1 \mathrm{H}$, br d, $J=15.6 \mathrm{~Hz}), 2.59(1 \mathrm{H}$, br t, $J=15.6 \mathrm{~Hz})$, $1.31\left(6 \mathrm{H}\right.$, br t, $\left.J=7.2 \mathrm{~Hz}, 2 \times \mathrm{OCH}_{2} \mathrm{CH}_{3}\right) ;{ }^{13} \mathrm{C} \mathrm{NMR}\left(\mathrm{CDCl}_{3}\right.$, DEPT) $\delta$ $200.9(\mathrm{C}, \mathrm{C}=\mathrm{O}), 169.4(\mathrm{C}, \mathrm{O}=\mathrm{C}-\mathrm{O}), 167.2(\mathrm{C}, \mathrm{O}=\mathrm{C}-\mathrm{O}), 147.7(\mathrm{C}), 146.3(\mathrm{C}), 126.6(2 \times \mathrm{CH}$, $\mathrm{Ph}-\mathrm{CH}), 123.9(2 \times \mathrm{CH}, \mathrm{Ph}-\mathrm{CH}), 82.1(\mathrm{C}, \mathrm{C}-2), 74.3(\mathrm{CH}, \mathrm{C}-6), 62.8\left(2 \times \mathrm{CH}_{2}, \mathrm{OCH}_{2} \mathrm{CH}_{3}\right)$, $47.5\left(\mathrm{CH}_{2}\right), 44.1\left(\mathrm{CH}_{2}\right), 13.93\left(\mathrm{CH}_{3}, \mathrm{OCH}_{2} \mathrm{CH}_{3}\right), 13.91\left(\mathrm{CH}_{3}, \mathrm{OCH}_{2} \mathrm{CH}_{3}\right) ; \mathrm{LRMS}: m / z 388.25$ $\left(\mathrm{M}+\mathrm{Na}^{+}\right)$, calcd for $\mathrm{C}_{17} \mathrm{H}_{19} \mathrm{NO}_{8} \mathrm{Na}^{+} 388.1008$.

2-Hydroxy-2-[4-(4-nitrophenyl)-2-oxobut-3-enyl]malonic acid diethyl ester (7p). Purified by column chromatography using EtOAc/hexane and isolated as oil. IR (neat): $v_{\max } 3651$ (O$\mathrm{H}), 3078,2984$, 2938, $1742(\mathrm{C}=\mathrm{O}, \mathrm{O}-\mathrm{C}=\mathrm{O}), 1603,1524,1468,1348,1290,1227,1165$, 1072, 1014, 856, 748, 698, $646 \mathrm{~cm}^{-1} ;{ }^{1} \mathrm{H}$ NMR $\left(\mathrm{CDCl}_{3}\right) \delta 8.26(2 \mathrm{H}, \mathrm{d}, J=8.4 \mathrm{~Hz}), 7.72(2 \mathrm{H}$, 


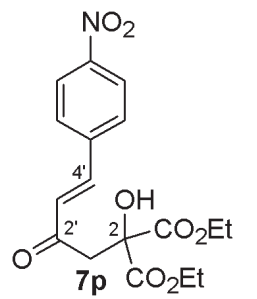

$\mathrm{d}, J=8.8 \mathrm{~Hz})[\mathrm{Ph}-H] ; 7.61(1 \mathrm{H}, \mathrm{d}, J=16.4 \mathrm{~Hz}$, olefinic- $\beta-\mathrm{H}), 6.84(1 \mathrm{H}, \mathrm{d}, J$ $=16.4 \mathrm{~Hz}$, olefinic- $\alpha-\mathrm{H}), 4.34-4.26\left(4 \mathrm{H}, \mathrm{m}, 2 \times \mathrm{OCH}_{2} \mathrm{CH}_{3}\right), 3.56(2 \mathrm{H}, \mathrm{s}$, $\left.\mathrm{CH}_{2}\right), 1.30\left(6 \mathrm{H}\right.$, br t, $\left.J=7.2 \mathrm{~Hz}, 2 \times \mathrm{OCH}_{2} \mathrm{CH}_{3}\right) ;{ }^{13} \mathrm{C} \mathrm{NMR}\left(\mathrm{CDCl}_{3}, \mathrm{DEPT}\right) \delta$ $195.5(\mathrm{C}, \mathrm{C}=\mathrm{O}), 166.6(2 \times \mathrm{C}, \mathrm{O}=\mathrm{C}-\mathrm{O}), 148.7(\mathrm{C}), 140.7(\mathrm{CH}$, olefinic- $\beta$ $\mathrm{CH}), 140.2(\mathrm{C}), 129.1(\mathrm{CH}$, olefinic- $\alpha-\mathrm{CH}), 128.9(2 \times \mathrm{CH}, \mathrm{Ph}-\mathrm{CH}), 124.1(2$ $\times \mathrm{CH}, \mathrm{Ph}-\mathrm{CH}), 76.6(\mathrm{C}, \mathrm{C}-\mathrm{OH}), 62.8\left(2 \times \mathrm{CH}_{2}, \mathrm{OCH}_{2} \mathrm{CH}_{3}\right), 45.4\left(\mathrm{CH}_{2}\right)$, $13.91\left(2 \times \mathrm{CH}_{3}, \mathrm{OCH}_{2} \mathrm{CH}_{3}\right)$; LRMS: $\boldsymbol{m} / z, 388.25\left(\mathrm{M}+\mathrm{Na}^{+}\right)$, calcd for $\mathrm{C}_{17} \mathrm{H}_{19} \mathrm{NO}_{8} \mathrm{Na}^{+} 388.1008$.

\section{Acknowledgments}

We thank DST, New Delhi (DBR and RM) and UGC Networking Resource Centre (DBR and SJ) for support of this project. RM thanks CSIR (New Delhi) for her research fellowship.

\section{Supporting information available}

Experimental procedures, compound characterization, and analytical data $\left({ }^{1} \mathrm{H} \mathrm{NMR},{ }^{13} \mathrm{C}\right.$ NMR and HRMS) for all new compounds. This material is available on the WWW at http://www.arkat-usa.org/get-file/54850/ or from the author.

\section{References}

1. Gouverneur, V.; Reiter, M. Chem. Eur. J. 2005, 11, 5806-5815.

http://dx.doi.org/10.1002/chem.200500406

2. Jørgensen, K. A. Angew. Chem. Int. Ed. 2000, 39, 3558-3588.

http://dx.doi.org/10.1002/1521-3773(20001016)39:20<3558::AID-ANIE3558>3.0.CO;2-I

3. Kagan, H. B.; Riant, O. Chem. Rev. 1992, 92, 1007-1019. http://dx.doi.org/10.1021/cr00013a013

4. Maruoka, K.; Yamamoto, H. J. Am. Chem. Soc. 1989, 111, 789-790. http://dx.doi.org/10.1021/ja00184a087

5. Schaus, S. E.; Branalt, J.; Jacobsen, E. N. J. Org. Chem. 1998, 63, 403-405. http://dx.doi.org/10.1021/j0971758c

6. Bednarski, M.; Danishefsky, S. J. Am. Chem. Soc. 1983, 105, 6968-6989. http://dx.doi.org/10.1021/ja00349a064

7. Keck, G. E.; Li, X-Y.; Krishnamurthy, D. J. Org. Chem. 1995, 60, 5998-5999. http://dx.doi.org/10.1021/jo00124a001

8. Notz, W.; Tanaka, F.; Barbas III, C. F. Acc. Chem. Res. 2004, 37, 580-591. http://dx.doi.org/10.1021/ar0300468 
9. Dalko, P. I.; Moisan, L. Angew. Chem. Int. Ed. 2001, 40, 3726.

http://dx.doi.org/10.1002/1521-3773(20011015)40:20<3726::AID-ANIE3726>3.0.CO;2-D

10. Schreiner, P. R. Chem. Soc. Rev. 2003, 32, 289-296.

http://dx.doi.org/10.1039/b107298f

11. Huang, Y.; Rawal, V. H. J. Am. Chem. Soc. 2002, 124, 9662-9663.

http://dx.doi.org/10.1021/ja0267627

12. Huang, Y.; Unni, A. K.; Thadani, A. N.; Rawal, V. H. Nature 2003, 424, 146. http://dx.doi.org/10.1038/nature01745

13. Unni, A. K.; Takenaka, N.; Yamamoto, H.; Rawal, V. H. J. Am. Chem. Soc. 2005, 127, $1336-1337$.

http://dx.doi.org/10.1021/ja044076x

14. Juhl, K.; Jørgensen, K. A. Angew. Chem. Int. Ed. 2003, 42, 1498-1501.

http://dx.doi.org/10.1002/anie.200250652

15. Yamamoto, Y.; Momiyama, N.; Yamamoto, H. J. Am. Chem. Soc. 2004, 126, 5962-5963. http://dx.doi.org/10.1021/ja049741g

16. Hayashi, Y.; Yamaguchi, J.; Hibino, K.; Sumiya, T.; Urushima, T.; Shoji, M.; Hashizume, D.; Koshino, H. Adv. Synth. Catal. 2004, 346, 1435-1439.

http://dx.doi.org/10.1002/adsc.200404166

17. Sundén, H.; Dahlin, N.; Ibrahem, I.; Adolfsson, H.; Córdova, A. Tetrahedron Lett. 2005, 46, 3385-3389.

http://dx.doi.org/10.1016/j.tetlet.2005.03.085

18. Sundén, H.; Ibrahem, I.; Eriksson, L.; Córdova, A. Angew. Chem. Int. Ed. 2005, 44, 48774880 .

http://dx.doi.org/10.1002/anie.200500811

19. Ramachary, D. B.; Chowdari, N. S.; Barbas III, C. F. Angew. Chem. Int. Ed. 2003, 42, 4233-4237.

http://dx.doi.org/10.1002/anie.200351916

20. Ramachary, D. B.; Anebouselvy, K.; Chowdari, N. S.; Barbas III, C. F. J. Org. Chem. 2004, 69, 5838-5849.

http://dx.doi.org/10.1021/jo049581r

21. Ramachary, D. B.; Barbas III, C. F. Chem. Eur. J. 2004, 10, 5323-5331.

http://dx.doi.org/10.1002/chem.200400597

22. Ramachary, D. B.; Barbas III, C. F. Org. Lett. 2005, 7, 1577-1580.

http://dx.doi.org/10.1021/o1050246e

23. Ramachary, D. B.; Ramakumar, K.; Kishor, M. Tetrahedron Lett. 2005, 46, 7037-7042. http://dx.doi.org/10.1016/j.tetlet.2005.08.051

24. Ramachary, D. B.; Kishor, M.; Ramakumar, K. Tetrahedron Lett. 2006, 47, 651-656. http://dx.doi.org/10.1016/j.tetlet.2005.11.128

25. Steiner, D. D.; Mase, N.; Barbas III, C. F. Angew. Chem. Int. Ed. 2005, 44, 3706-3710. http://dx.doi.org/10.1002/anie.200500571

26. Suri, J. T.; Ramachary, D. B.; Barbas III, C. F. Org. Lett. 2005, 7, 1383-1385.

http://dx.doi.org/10.1021/o10502533 
27. Ramachary, D. B.; Reddy, Y. V. Eur. J. Org. Chem. 2012, 865-887. http://dx.doi.org/10.1002/ejoc.201101157

28. Yin, J.; Kouda, K.; Tezuka, Y.; Tran, Q. L.; Miyahara, T.; Chen, Y.; Kadota, S. Planta Med. 2004, 70, 54-58.

http://dx.doi.org/10.1055/s-2004-815456

29. Chandrasekhar, S.; Shyamsunder, T.; Jaya Prakash, S.; Prabhakar, A.; Jagadeesh, B. Tetrahedron Lett. 2006, 47, 47-49.

http://dx.doi.org/10.1016/j.tetlet.2005.10.129

30. Trost, B. M.; Yang, H.; Wuitschik, G. Org. Lett. 2005, 7, 4761-4764.

http://dx.doi.org/10.1021/o10520065 